hep-ph/9511403

DPNU-95-37

TUIMP-TH-95/71

\title{
Four-Body Chiral Order Parameters in the Massless Fermion Phase
}

\author{
Pieter Maris \\ Department of Physics, Nagoya University, Nagoya 464-01, Japan \\ Qing Wang \\ Department of Physics, Tsinghua University, Beijing 100084, China
}

(November 15, 1995)

\begin{abstract}
The fermion four-point functions and condensates as the chiral symmetry order parameters are calculated analytically in U(1) gauge theory in the massless phase. It is shown that in leading order of the loop expansion of the effective action, there is a critical coupling for the nonperturbative parity-invariant chirality-changing four-fermion functions; this critical coupling however is the same as that for dynamical mass generation. Therefore there is no breaking of chiral symmetry through the condensation of four-fermion functions below the critical coupling for dynamical mass generation for the fermions.
\end{abstract}

11.15.Tk, 11.30.Qc, 11.30.Rd

Typeset using REVTEX 


\section{INTRODUCTION}

Dynamical chiral symmetry breaking (DCSB) plays a very important role in the research of fundamental interactions. Up to now, most investigations are focussed on DCSB with mass generation for the fermions. In such a scenario, the bilinear fermion condensate is taken as the order parameter of chiral symmetry breaking and it is known that it can receive a nonzero value when the coupling of the system exceeds some critical value. For a review on dynamical chiral symmetry breaking, see e.g. [1].

But this is not the complete story for DCSB, since the order parameter may not be the bilinear fermion condensate: in principle, higher $n$-point functions which break chiral symmetry might become nonzero, without dynamical mass generation for the fermions [2, 3]. In a massless $U(1)$ gauge theory for example, four-fermion Green's functions which break chiral symmetry might become nonzero at values of the coupling below the critical value for dynamical mass generation. Such a breaking of the chiral symmetry by the four-fermion condensates, without mass generation for the fermions, was discussed recently by Holdom and Triantaphyllou [2], who have calculated these four-point functions numerically and found a critical coupling constant which is almost the same as that responsible for dynamical mass generation.

One could also think of theories where some other symmetry prevents the dynamical generation of a bilinear condensate and a dynamical fermion mass, but where a nonzero condensate of a higher $n$-point function is not restricted by this symmetry. In a chiral gauge theory, the gauge symmetry will be broken by the generation of a fermion mass, but a chirality-changing four-fermion condensate does not break the gauge symmetry, and might therefore be generated dynamically. In such a scenario, the chiral symmetry can be realized in more delicate way which may be useful for model building [4 6]: it allows for a hierarchy of chiral symmetry breaking, first by the four-fermion condensate at relatively small values of the coupling, and eventually a second phase transition, at higher values of the coupling, leading to mass generation for the fermions, due to the four-fermion condensates. For these purposes one should study this phenomenon in a chiral gauge theory, as suggested in [6], but for simplicity and to see whether such a symmetry breaking patterns is possible, we restrict ourselves to a $U(1)$ gauge theory.

In our paper we confirm analytically the numerical results obtained by Holdom and Triantaphyllou [2], using a slightly different method to calculate the nonperturbative fourfermion functions. We find that there is a critical coupling for dynamical chiral symmetry breaking via the four-fermion condensates, below which there are no nontrivial solutions for the four-fermion functions which break chiral symmetry, but preserve parity, Lorentz symmetry, and the flavor symmetry. This critical coupling is (in our truncation scheme) exactly the same as the critical coupling for fermion mass generation. This result is obtained by calculating the loop expansion of the effective action to leading order for the nonperturbative four-point functions. Our result is also in agreement with an intuitive argument comparing the condensation of two fermions to the condensation of four fermions: for the four-fermion condensate we need at least the same binding force as for the bilinear fermion condensate.

Our paper is organized as follows. In the next section, we discuss some general features about dynamical symmetry breaking, and we show how in principle the chiral symmetry can

be broken without mass generation for the fermions. In Sec. [II, we derive a linear integral 
equation for the four-fermion function, using the loop expansion of the effective action. Next, we analyze this equation for the nonperturbative chirality-changing four-fermion functions, using some expansions in order to perform the angular integrations analytically; more details can be found in the appendix. We find that there is a critical coupling for these functions; this critical coupling turns out to be the same as the critical coupling for dynamical mass generation. Finally we give some concluding remarks in Sec. \.

\section{GENERAL CONSIDERATIONS ON DYNAMICAL SYMMETRY BREAKING}

\section{A. Symmetry Breaking Patterns}

The kind of symmetry breaking we are dealing with in this paper is the breaking of chiral symmetry in a $U(1)$ gauge theory with $N_{f}$ massless fermion flavors. The global continuous chiral symmetry is $U_{L}\left(N_{f}\right) \otimes U_{R}\left(N_{f}\right)$, and this symmetry can be broken in different ways, characterized by the corresponding chiral order parameters. Since the flavor symmetry is not broken in vector-like gauge theories [7], only flavor-symmetry invariant order parameters may receive nonzero contributions; also parity [8] and Lorentz symmetry is believed not to be broken spontaneously, so in this paper we limit us to parity-, flavor-, and Lorentz-invariant order parameters.

The simplest and most wellknown way of breaking the chiral symmetry is characterized by the trace of the two-point function $\langle 0|\mathbf{T} \bar{\psi}(x) \psi(y)| 0\rangle$. This condensate, $\langle 0|\bar{\psi}(x) \psi(x)| 0\rangle$, is the only possible two-fermion condensate which keeps the flavor symmetry and is parity and Lorentz invariant. It gives rise to a dynamically generated fermion mass, and the symmetry breaking pattern is

$$
U_{L}\left(N_{f}\right) \otimes U_{R}\left(N_{f}\right) \longrightarrow U_{L+R}\left(N_{f}\right)
$$

An explicit mass for the fermions would also break the symmetry in this way.

However, this is not the only way to break the chiral symmetry, an alternative way is characterized by condensates of four fermions. The possible four-point function which keep flavor and Lorentz symmetry and which are parity invariant are

$$
\begin{aligned}
\mathcal{G}_{0, S+P}\left(x_{1}, x_{2}, x_{3}, x_{4}\right)= & \left\langle 0\left|\mathbf{T} \bar{\psi}\left(x_{1}\right) \lambda^{0} \psi\left(x_{2}\right) \bar{\psi}\left(x_{3}\right) \lambda^{0} \psi\left(x_{4}\right)\right| 0\right\rangle \\
& +\left\langle 0\left|\mathbf{T} \bar{\psi}\left(x_{1}\right) \gamma_{5} \lambda^{0} \psi\left(x_{2}\right) \bar{\psi}\left(x_{3}\right) \gamma_{5} \lambda^{0} \psi\left(x_{4}\right)\right| 0\right\rangle, \\
\mathcal{G}_{S+P}\left(x_{1}, x_{2}, x_{3}, x_{4}\right)= & \sum_{\alpha=1}^{N_{f}^{2}-1}\left[\left\langle 0\left|\mathbf{T} \bar{\psi}\left(x_{1}\right) \lambda^{\alpha} \psi\left(x_{2}\right) \bar{\psi}\left(x_{3}\right) \lambda^{\alpha} \psi\left(x_{4}\right)\right| 0\right\rangle\right. \\
& \left.+\left\langle 0\left|\mathbf{T} \bar{\psi}\left(x_{1}\right) \gamma_{5} \lambda^{\alpha} \psi\left(x_{2}\right) \bar{\psi}\left(x_{3}\right) \gamma_{5} \lambda^{\alpha} \psi\left(x_{4}\right)\right| 0\right\rangle\right], \\
\mathcal{G}_{0, T}\left(x_{1}, x_{2}, x_{3}, x_{4}\right)= & \left\langle 0\left|\mathbf{T} \bar{\psi}\left(x_{1}\right) \sigma_{\mu \nu} \lambda^{0} \psi\left(x_{2}\right) \bar{\psi}\left(x_{3}\right) \sigma^{\mu \nu} \lambda^{0} \psi\left(x_{4}\right)\right| 0\right\rangle, \\
& \mathcal{G}_{f}^{2}-1 \\
\mathcal{G}_{T}\left(x_{1}, x_{2}, x_{3}, x_{4}\right)= & \sum_{\alpha=1}\left\langle 0\left|\mathbf{T} \bar{\psi}\left(x_{1}\right) \sigma_{\mu \nu} \lambda^{\alpha} \psi\left(x_{2}\right) \bar{\psi}\left(x_{3}\right) \sigma^{\mu \nu} \lambda^{\alpha} \psi\left(x_{4}\right)\right| 0\right\rangle .
\end{aligned}
$$

In these expressions, $\lambda^{\alpha}$ is the flavor matrix with $\alpha=1, \ldots, N_{f}^{2}-1 ; \lambda^{0}$ is proportional to the unit matrix. These four-fermion functions can be related to the axial charge, and 
the condensate of these four-fermion functions (with all the coordinates $x_{k}$ in the same space-time point) can be considered as the order parameters of DCSB.

A nonzero condensate of (one of) these four-fermion functions gives the symmetry breaking pattern

$$
U_{L}\left(N_{f}\right) \otimes U_{R}\left(N_{f}\right) \longrightarrow U_{L+R}\left(N_{f}\right) \otimes Z_{4}
$$

Here $Z_{4}$ is a nontrivial chiral subgroup which plays a role in preventing dynamical mass generation for the fermions. It corresponds to the discrete chiral transformation

$$
\psi(x) \rightarrow \mathrm{e}^{i \frac{k}{2} \pi \gamma_{5}} \psi(x)
$$

and therefore the fermions remain massless in such a breaking of the chiral symmetry.

Note that in principle there are a lot more four-fermion functions which break the chiral symmetry. In the case of one fermion flavor, $N_{f}=1$, there are in total 54 independent functions which all lead to the same chiral symmetry breaking pattern, Eq. (2.6), but the ones above, which we will call $S+P$ and $T$ type four-fermion functions, are the only ones which preserve parity and Lorentz symmetry. The other 52 independent functions are not all parity-invariant or Lorentz scalars, which means they will not receive nonzero condensates, assuming that the Lorentz symmetry and parity remains unbroken. However, it is not excluded that (some of) the nonlocal four-fermion functions become nonzero, in which case they might couple to the $S+P$ and $T$ type functions. We leave this problem to future investigations and for simplicity we only consider the nonperturbative four-point functions which are flavor-, Lorentz- and parity-invariant.

This scenario can easily be generalized to condensates of $2 n$ fermions, breaking the chiral symmetry into

$$
U_{L}\left(N_{f}\right) \otimes U_{R}\left(N_{f}\right) \longrightarrow U_{L+R}\left(N_{f}\right) \otimes Z_{2 n}
$$

with the nontrivial chiral subgroup $Z_{2 n}$, corresponding to the discrete chiral transformation

$$
\psi(x) \rightarrow \mathrm{e}^{i \frac{k}{n} \pi \gamma_{5}} \psi(x)
$$

unbroken.

\section{B. Goldstone Bosons}

It is wellknown that if a continuous symmetry of the Lagrangian is broken dynamically, there will appear some massless bosons, the socalled Goldstone or Nambu-Goldstone bosons [9.10]. These Goldstone bosons couple both to the conserved current which is associated with the symmetry, and to some fields $\phi$ which characterize the breaking of the symmetry. Note that these fields $\phi$, which can be regarded as the order parameters of the phase transition, can be either composite or elementary.

In the case of dynamical breaking of chiral symmetry, the Goldstone bosons are related to the chiral-symmetry breaking $n$-point functions through the axial (or chiral) WardTakahashi identity. The conserved chiral current is 


$$
j_{5}^{\mu}(x)=\bar{\psi}(x) \gamma_{5} \gamma^{\mu} \psi(x)
$$

and we consider the following Green's function in momentum space

$$
G_{5}^{\mu}(q)=\int d^{4} x \mathrm{e}^{i q x}\left\langle 0\left|j_{5}^{\mu}(0) \phi(x)\right| 0\right\rangle,
$$

where $\phi$ is a (composite) field associated with the chiral order parameter we are considering】. The breaking of chiral symmetry means that this order parameter becomes nonzero, and due to the chiral Ward-Takahashi identity this leads to a pole at zero momentum in the above Green's function; in the limit $q_{\mu} \rightarrow 0$ this Green's function behaves as

$$
\begin{aligned}
G_{5}^{\mu}(q) & \sim \frac{q^{\mu}}{q^{2}} \int d x \mathrm{e}^{i q x} i \partial_{\nu}\left\langle 0\left|j_{5}^{\nu}(0) \phi(x)\right| 0\right\rangle \\
& \sim \frac{-q^{\mu}}{q^{2}}\langle 0|\delta \phi| 0\rangle .
\end{aligned}
$$

To be more specific, we first consider conventional chiral symmetry breaking, with a dynamically generated mass for the fermions. In that case, the composite field $\phi(x-y)$ is $\psi(x) \bar{\psi}(y)$, and the chiral Ward-Takahashi identity leads to

$$
(p-k)_{\mu} G_{5}^{\mu}(p-k)=S(p) \gamma_{5}+\gamma_{5} S(k),
$$

where $S(p)$ is the full fermion propagator. From this equation, we see immediately that a dynamically generated mass for the fermions will lead to a massless pole in the pseudoscalar Green's function $G_{5}^{\mu}(p-k)$ if $p \rightarrow k$; this pole corresponds to the massless Goldstone boson.

In the case of dynamical chiral symmetry breaking through the four-point functions, as we are considering here, the situation is somewhat more complicated since we have to consider a composite field $\phi$ which consists of four fermion fields. Using the chiral WardTakahashi identity, one can show that the Green's functions $G_{\mu}^{5}\left(q, p_{i}\right)$ have to satisfy the relation

$$
\begin{aligned}
q_{\mu}\left(G_{5}^{\mu}\left(q, p_{i}\right)\right)_{a b c d}= & \left(\gamma_{5}\right)_{a a^{\prime}} \mathcal{G}_{a^{\prime} b c d}\left(p_{1}+q, p_{2}, p_{3}, p_{4}\right)+\mathcal{G}_{a b^{\prime} c d}\left(p_{1}, p_{2}+q, p_{3}, p_{4}\right)\left(\gamma_{5}\right)_{b^{\prime} b} \\
& +\left(\gamma_{5}\right)_{c c^{\prime}} \mathcal{G}_{a b c^{\prime} d}\left(p_{1}, p_{2}, p_{3}+q, p_{4}\right)+\mathcal{G}_{a b c d^{\prime}}\left(p_{1}, p_{2}, p_{3}, p_{4}+q\right)\left(\gamma_{5}\right)_{d d^{\prime}},
\end{aligned}
$$

where $\mathcal{G}_{a b c d}\left(p_{1}, p_{2}, p_{3}, p_{4}\right)$ is the Fourier transform of a four-fermion function, with momentum conservation $\sum p_{i}=0$. In the limit $q_{\mu} \rightarrow 0$, this leads to massless poles, if either the $S+P$ type or the $T$ type four-fermion function

$$
\begin{aligned}
\mathcal{G}_{S+P}\left(p_{1}, p_{2}, p_{3}, p_{4}\right)= & \int d^{4} x_{1} \cdots d^{4} x_{4} \mathrm{e}^{-i \sum p_{i} x_{i}}\left\langle 0\left|\mathbf{T} \bar{\psi}\left(x_{1}\right) \psi\left(x_{2}\right) \bar{\psi}\left(x_{3}\right) \psi\left(x_{4}\right)\right| 0\right\rangle \\
& +\int d^{4} x_{1} \cdots d^{4} x_{4} \mathrm{e}^{-i \sum p_{i} x_{i}}\left\langle 0\left|\mathbf{T} \bar{\psi}\left(x_{1}\right) \gamma_{5} \psi\left(x_{2}\right) \bar{\psi}\left(x_{3}\right) \gamma_{5} \psi\left(x_{4}\right)\right| 0\right\rangle, \\
\mathcal{G}_{T}\left(p_{1}, p_{2}, p_{3}, p_{4}\right)= & \int d^{4} x_{1} \cdots d^{4} x_{4} \mathrm{e}^{-i \sum p_{i} x_{i}}\left\langle 0\left|\mathbf{T} \bar{\psi}\left(x_{1}\right) \sigma_{\mu \nu} \psi\left(x_{2}\right) \bar{\psi}\left(x_{3}\right) \sigma^{\mu \nu} \psi\left(x_{4}\right)\right| 0\right\rangle,
\end{aligned}
$$

\footnotetext{
${ }^{1}$ Here we only write down the space-time coordinate $x$ which is related to Goldstone pole in momentum space; in general, $\phi$ can also depend on other space-time coordinates, which do not relate to the Goldstone pole.
} 
is nonzero. This can be seen easily by decomposing the Green's function $G_{5}^{\mu}$ into

$$
G_{5}^{\mu}\left(q, p_{i}\right)=\frac{q_{\mu}}{q^{2}}\left(\chi_{S+P}\left(q, p_{i}\right)\left(I \otimes \gamma_{5}+\gamma_{5} \otimes I\right)+\chi_{T}\left(q, p_{i}\right)\left(\gamma_{5} \sigma_{\rho \sigma}\right) \otimes \sigma^{\rho \sigma}\right)+\ldots
$$

so Eq. (2.15) leads to

$$
\begin{aligned}
\chi_{S+P}\left(q, p_{i}\right)= & \mathcal{G}_{S+P}\left(p_{1}+q, p_{2}, p_{3}, p_{4}\right)+\mathcal{G}_{S+P}\left(p_{1}, p_{2}+q, p_{3}, p_{4}\right) \\
& +\mathcal{G}_{S+P}\left(p_{1}, p_{2}, p_{3}+q, p_{4}\right)+\mathcal{G}_{S+P}\left(p_{1}, p_{2}, p_{3}, p_{4}+q\right), \\
\chi_{T}\left(q, p_{i}\right)= & \mathcal{G}_{T}\left(p_{1}+q, p_{2}, p_{3}, p_{4}\right)+\mathcal{G}_{T}\left(p_{1}, p_{2}+q, p_{3}, p_{4}\right) \\
& +\mathcal{G}_{T}\left(p_{1}, p_{2}, p_{3}+q, p_{4}\right)+\mathcal{G}_{T}\left(p_{1}, p_{2}, p_{3}, p_{4}+q\right) .
\end{aligned}
$$

Thus massless Goldstone bosons appear as soon as any of these nonperturbative four-point functions becomes nonzero.

If we restrict ourselves to local composite fields $\phi$ we get a similar expression, but now involving the condensates of these four-point functions. Defining the condensate as

$$
\int \frac{d^{4} p_{1} d^{4} p_{2} d^{4} p_{3} d^{4} p_{4}}{(2 \pi)^{12}} \mathcal{G}_{i}\left(p_{1}, p_{2}, p_{3}, p_{4}\right) \delta\left(p_{1}+p_{2}+p_{3}+p_{4}\right)
$$

where $i$ denotes either $S+P$ or $T$, the chiral Ward-Takahashi identity relates this condensate to the Goldstone bosons. In momentum space this finally leads to massless poles for $q^{2} \rightarrow 0$

$$
\begin{gathered}
\chi_{S+P}(0)=4 \int \frac{d^{4} p_{1} d^{4} p_{2} d^{4} p_{3}}{(2 \pi)^{12}} \mathcal{G}_{S+P}\left(p_{1}, p_{2}, p_{3},-p_{1}-p_{2}-p_{3}\right), \\
\chi_{T}(0)=4 \int \frac{d^{4} p_{1} d^{4} p_{2} d^{4} p_{3}}{(2 \pi)^{12}} \mathcal{G}_{T}\left(p_{1}, p_{2}, p_{3},-p_{1}-p_{2}-p_{3}\right) .
\end{gathered}
$$

Thus the condensates of the four-point functions we are considering here are directly related to the existence of massless Goldstone bosons.

Note that both a nonzero condensate and a nonzero four-point function would signal dynamical chiral symmetry breaking, and in principle it is conceivable that the condensate is zero, and the four-point function not; the other way around (no nonzero four-point function, but only a nonzero condensate) is obviously impossible. In this paper, instead of fourfermion condensate, we directly calculate four-point function and take it as a generalized order parameter.

The generalization to the case of $N$ fermion flavors is in principle straightforward; also the generalization to higher $n$-point functions is a trivial exercise.

\section{EFFECTIVE ACTION}

In order to calculate the chirality-changing four-fermion functions, we need some nonperturbative calculation scheme. For this purpose, we use a loop expansion of the effective action [11]13. For simplicity we will restrict ourselves to just one fermion flavor, so the generating functional in Minkowski space is

$$
Z[\bar{I}, I, J]=\mathrm{e}^{W[\bar{I}, I, J]}=\int \mathcal{D} \bar{\psi} \mathcal{D} \psi \mathcal{D} A_{\mu} \mathrm{e}^{i \int d^{4} x\left[\mathcal{L}(A)+\bar{\psi}(i \not \partial-e \not A) \psi+\bar{I} \psi+\bar{\psi} I+J_{\mu} A^{\mu}\right]},
$$


where $\mathcal{L}(A)$ is the Lagrangian for the photon field

$$
\mathcal{L}(A)=-\frac{1}{4} F_{\mu \nu} F^{\mu \nu}-\frac{1}{2 a}\left(\partial_{\mu} A^{\mu}\right)^{2},
$$

in a general covariant gauge. In order to calculate the effective action, we start by integrating out the photon field, which gives

$$
\begin{aligned}
& \int \mathcal{D} A_{\mu} \mathrm{e}^{i \int d^{4} x\left[\mathcal{L}(A)+\left(-e \bar{\psi} \gamma^{\mu} \psi+J^{\mu}\right) A_{\mu}\right]}= \\
& =\exp \left[\frac{-i}{2} \int d^{4} x d^{4} y\left(-e \bar{\psi}(x) \gamma^{\mu} \psi(x)+J^{\mu}(x)\right) D_{\mu \nu}(x, y)\left(-e \bar{\psi}(y) \gamma^{\nu} \psi(y)+J^{\nu}(y)\right)\right]
\end{aligned}
$$

where $D_{\mu \nu}(x, y)$ is the bare photon propagator

$$
D_{\mu \nu}(x, y) \equiv \frac{1}{\partial^{2}}\left[g_{\mu \nu}-\frac{\partial_{\mu} \partial_{\nu}}{\partial^{2}}(1+a)\right] \delta^{4}(x-y),
$$

in which the derivative $\partial_{\mu}$ is acting on the coordinate $x$, and $a$ is the gauge-fixing parameter.

Next, we introduce a generalized Green's function containing four space-time points, with spinor indices $a, b, c$, and $d$

$$
\bar{D}_{c d}^{a b}\left(x, x^{\prime}, y, y^{\prime}\right) \equiv D_{\mu \nu}(x, y) \gamma_{a d}^{\mu} \gamma_{b c}^{\nu} \delta^{4}\left(x^{\prime}-y\right) \delta^{4}\left(y^{\prime}-x\right)
$$

such that

$$
\begin{aligned}
& -D_{\mu \nu}(x, y)\left(\bar{\psi}(x) \gamma^{\mu} \psi(x)\right)_{a a}\left(\bar{\psi}(y) \gamma^{\nu} \psi(y)\right)_{b b}= \\
& =\int d^{4} x^{\prime} d^{4} y^{\prime} \bar{D}_{c d}^{a b}\left(x, x^{\prime}, y, y^{\prime}\right) \bar{\psi}^{a}(x) \psi^{c}\left(x^{\prime}\right) \bar{\psi}^{b}(y) \psi^{d}\left(y^{\prime}\right) .
\end{aligned}
$$

Note that in the case with $N_{f}$ fermion flavors, we just have to add additional indices to label the different flavors. Thus the generating functional can be written as

$$
\begin{aligned}
Z[\bar{I}, I, J]= & \int \mathcal{D} \psi \mathcal{D} \bar{\psi} \exp i\left[\int d^{4} x(\bar{\psi} i \not \partial \psi+\bar{I} \psi+\bar{\psi} I)\right. \\
& +\frac{e^{2}}{2} \int d^{4} x d^{4} x^{\prime} d^{4} y d^{4} y^{\prime} \bar{D}_{c d}^{a b}\left(x, x^{\prime}, y, y^{\prime}\right) \bar{\psi}^{a}(x) \psi^{c}\left(x^{\prime}\right) \bar{\psi}^{b}(y) \psi^{d}\left(y^{\prime}\right) \\
& \left.-\frac{1}{2} \int d^{4} x d^{4} y D_{\mu \nu}(x, y)\left(-2 e \bar{\psi}(x) \gamma^{\mu} \psi(x) J^{\nu}(y)+J^{\mu}(x) J^{\nu}(y)\right)\right]
\end{aligned}
$$

Inserting a Gaussian integral of a bilocal auxiliary field $\chi_{a b}\left(x, x^{\prime}\right)$

$$
\begin{aligned}
\text { const }= & \int \mathcal{D} \chi \exp \frac{-i}{2}\left[\int d^{4} x d^{4} x^{\prime} d^{4} y d^{4} y^{\prime} \bar{D}_{c d}^{a b}\left(x, x^{\prime}, y, y^{\prime}\right) \times\right. \\
& \left.\left(\chi^{a c}\left(x, x^{\prime}\right)-e \bar{\psi}^{a}(x) \psi^{c}\left(x^{\prime}\right)\right)\left(\chi^{b d}\left(y, y^{\prime}\right)-e \bar{\psi}^{b}(y) \psi^{d}\left(y^{\prime}\right)\right)\right],
\end{aligned}
$$

gives for the generating functional 


$$
\begin{aligned}
& Z[\bar{I}, I, J]=\int \mathcal{D} \chi \mathcal{D} \psi \mathcal{D} \bar{\psi} \exp i\left[\int d^{4} x\left(\bar{\psi}(x) i \not x_{x} \psi(x)+\bar{I}(x) \psi(x)+\bar{\psi}(x) I(x)\right)\right. \\
& +\int d^{4} x d^{4} x^{\prime} d^{4} y d^{4} y^{\prime} \bar{D}_{c d}^{a b}\left(x, x^{\prime}, y, y^{\prime}\right)\left(e \bar{\psi}^{a}(x) \chi^{b d}\left(y, y^{\prime}\right) \psi^{c}\left(x^{\prime}\right)-\frac{1}{2} \chi^{a c}\left(x, x^{\prime}\right) \chi^{b d}\left(y, y^{\prime}\right)\right) \\
& \left.+e \int d^{4} x d^{4} y \bar{\psi}(x) \gamma^{\mu} D_{\mu \nu}(x, y) J^{\nu}(y) \psi(x)-\frac{1}{2} \int d^{4} x d^{4} y J^{\mu}(x) D_{\mu \nu}(x, y) J^{\nu}(y)\right]
\end{aligned}
$$

We see that the fermion field appears quadratically in the generating functional, so that they can be easily be integrated out. Thus we arrive at

$$
\begin{aligned}
Z[\bar{I}, I, J]= & \int \mathcal{D} \chi \mathrm{e}^{i S_{\mathrm{eff}}[\chi, \bar{I}, I, J]} \\
= & \int \mathcal{D} \chi \exp i\left[\frac{-1}{2} \int d^{4} x d^{4} x^{\prime} d^{4} y d^{4} y^{\prime} \bar{D}_{c d}^{a b}\left(x, x^{\prime}, y, y^{\prime}\right) \chi^{a c}\left(x, x^{\prime}\right) \chi^{b d}\left(y, y^{\prime}\right)\right. \\
& \left.+i \operatorname{Tr} \ln S-\int d^{4} x d^{4} y\left(\frac{1}{2} J^{\mu}(x) D_{\mu \nu}(x, y) J^{\nu}(y)+\bar{I}^{a}(x) S_{a b}(x, y) I^{b}(y)\right)\right]
\end{aligned}
$$

in which $S_{a b}(x, y)$ is defined as

$$
\begin{aligned}
& S_{a c}^{-1}\left(x, x^{\prime}\right) \equiv \\
& \equiv\left(i \not \partial_{x}+e \gamma^{\mu} \int d^{4} y D_{\mu \nu}(x, y) J^{\nu}(y)\right)_{a c} \delta^{4}\left(x-x^{\prime}\right)+e \int d^{4} y d^{4} y^{\prime} \bar{D}_{c d}^{a b}\left(x, x^{\prime}, y, y^{\prime}\right) \chi^{b d}\left(y, y^{\prime}\right) \\
& =\left(i \not \partial_{x}+e \gamma^{\mu} \int d^{4} y D_{\mu \nu}(x, y) J^{\nu}(y)\right)_{a c} \delta^{4}\left(x-x^{\prime}\right)+e D_{\mu \nu}\left(x, x^{\prime}\right) \gamma_{a d}^{\mu} \gamma_{b c}^{\nu} \chi^{b d}\left(x^{\prime}, x\right)
\end{aligned}
$$

The effective action for the $\chi$-field, induced by Eq. (3.10) and with external sources, is then given by

$$
\begin{aligned}
& S_{\mathrm{eff}}[\chi, \bar{I}, I, J]=\frac{-1}{2} \int d^{4} x d^{4} x^{\prime} D_{\mu \nu}\left(x, x^{\prime}\right) \gamma_{a d}^{\mu} \gamma_{b c}^{\nu} \chi^{a c}\left(x, x^{\prime}\right) \chi^{b d}\left(x^{\prime}, x\right) \\
& +i \operatorname{Tr} \ln S-\int d^{4} x d^{4} y\left(\frac{1}{2} J^{\mu}(x) D_{\mu \nu}(x, y) J^{\nu}(y)+\bar{I}^{a}(x) S_{a b}(x, y) I^{b}(y)\right)
\end{aligned}
$$

where we have used Eq. (3.5). Given the effective action $S_{\text {eff }}[\chi, \bar{I}, I, J]$ in Eq. (3.12), the calculation of various physical quantities concerns the calculation of the following generating functional

$$
\begin{aligned}
\tilde{Z}[\bar{I}, I, J, \tilde{J}] & =\mathrm{e}^{i \tilde{W}[\bar{I}, I, J, \tilde{J}]} \\
& =\int \mathcal{D} \chi \exp i\left[S_{\operatorname{eff}}[\chi, \bar{I}, I, J]+\int d^{4} X \quad \tilde{J}^{B}(X) \chi_{B}(X)\right],
\end{aligned}
$$

where $\tilde{J}^{B}(X)$ is an external source coupled to the $\chi_{B}(X)$ field, and we use a shorthand notation $X$ and $B$ for the bilocal variables $\left(x, x^{\prime}\right)$ and indices $(a b)$. The generating functional $Z[\bar{I}, I, J]$ in Eq. (3.8) is related to $\tilde{Z}[\bar{I}, I, J, \tilde{J}]$ by $Z[\bar{I}, I, J]=\tilde{Z}[\bar{I}, I, J, \tilde{J}=0]$. Now we define the classical field $\hat{\chi}_{B}(X)$ by

$$
\hat{\chi}_{B}(X) \equiv \frac{\delta \tilde{W}[\bar{I}, I, J, \tilde{J}]}{\delta \tilde{J}^{B}(X)},
$$


and the partial Legendre transformation

$$
\tilde{\Gamma}[\bar{I}, I, J, \hat{\chi}]=W[\bar{I}, I, J, \tilde{J}]-\int d^{4} X \quad \tilde{J}^{B}(X) \hat{\chi}_{B}(X) .
$$

It is easy to see that

$$
\frac{\delta \tilde{\Gamma}[\bar{I}, I, J, \hat{\chi}]}{\delta \hat{\chi}_{B}(X)}=-\tilde{J}^{B}(X)
$$

and hence

$$
W[\bar{I}, I, J]=\tilde{W}[\bar{I}, I, J, 0]=\left.\tilde{\Gamma}[\bar{I}, I, J, \hat{\chi}]\right|_{\frac{\delta \tilde{\Gamma}}{\delta \bar{\chi}_{B}}=0}
$$

Therefore what we need to do is to calculate $\tilde{\Gamma}[\bar{I}, I, J, \hat{\chi}]$. When we turn off the source for the bilocal field, $\tilde{J}^{B}(X)$, Eq. (3.16) becomes

$$
\frac{\delta \tilde{\Gamma}[\bar{I}, I, J, \hat{\chi}]}{\delta \hat{\chi}_{B}}=0
$$

which reflects the stability of the physical vacuum. The standard loop expansion formula for $\tilde{\Gamma}[\bar{I}, I, J, \hat{\chi}]$ is given in [12], and reduces in the present case to

$$
\tilde{\Gamma}[\bar{I}, I, J, \hat{\chi}]=S_{\text {eff }}[\hat{\chi}, \bar{I}, I, J]+\frac{i}{2} \operatorname{Tr} \ln \left[\frac{\delta^{2} S_{\text {eff }}[\hat{\chi}, \bar{I}, I, J]}{\delta \hat{\chi}_{B_{1}}\left(X_{1}\right) \delta \hat{\chi}_{B_{2}}\left(X_{2}\right)}\right]+\tilde{\Gamma}_{2},
$$

where the first term is the tree level contribution, the second term is the one-loop contribution, and $\tilde{\Gamma}_{2}$ represent higher-loop contributions, containing the sum over all higher loop 1 PI vacuum diagrams with the propagator

$$
\frac{\delta^{2} S_{\mathrm{eff}}[\hat{\chi}, \bar{I}, I, J]}{\delta \hat{\chi}_{B_{1}}\left(X_{1}\right) \delta \hat{\chi}_{B_{2}}\left(X_{2}\right)},
$$

and vertices

$$
\frac{\delta^{n} S_{\text {eff }}[\hat{\chi}, \bar{I}, I, J]}{\delta \hat{\chi}_{B_{1}}\left(X_{1}\right) \ldots \delta \hat{\chi}_{B_{n}}\left(X_{n}\right)} \quad(n \geq 3) .
$$

This expansion, Eq. (3.19), gives systematic rules to calculate the effective action, and we base our further discussion on this expansion. For simplicity, we consider the effective potential up to tree-level order only in our present paper, but in principle it is straightforward to go beyond this leading order calculation. At tree level, when the external source is switched off, we find

$$
W[\bar{I}, I, J]=S_{\text {eff }}[\hat{\chi}, \bar{I}, I, J]
$$

at the stationary point of the effective action

$$
\frac{\delta S_{\mathrm{eff}}[\hat{\chi}, \bar{I}, I, J]}{\delta \hat{\chi}^{a c}(x, y)}=0 .
$$


Using the definitions Eqs. (3.12) and (3.11), the stationary condition for the effective action, Eq. (3.23), leads to

$$
\hat{\chi}^{a c}\left(x, x^{\prime}\right)=-i e S^{c a}\left(x^{\prime}, x\right)-e \int d^{4} y d^{4} y^{\prime} S^{c b}\left(x^{\prime}, y\right) I^{b}(y) \bar{I}^{d}\left(y^{\prime}\right) S^{d a}\left(y^{\prime}, x\right) .
$$

Combined with Eq. (3.11), we find

$$
\begin{aligned}
& S_{a c}^{-1}\left(x, x^{\prime}\right)=\left(i \not \partial_{x}\right)_{a c} \delta^{4}\left(x-x^{\prime}\right)+e \gamma_{a c}^{\mu} \delta^{4}\left(x-x^{\prime}\right) \int d^{4} y D_{\mu \nu}(x, y) J^{\nu}(y) \\
& -e^{2} D_{\mu \nu}\left(x, x^{\prime}\right) \gamma_{a d}^{\mu} \gamma_{b c}^{\nu}\left[i S^{d b}\left(x, x^{\prime}\right)+\int d^{4} y d^{4} y^{\prime} S^{d a^{\prime}}\left(x, y^{\prime}\right) I^{a^{\prime}}\left(y^{\prime}\right) \bar{I}^{c^{\prime}}(y) S^{c^{\prime} b}\left(y, x^{\prime}\right)\right] .
\end{aligned}
$$

Note from Eq. (3.22) and Eqs. (3.12) and (3.23), that the function $S$ is related to the full fermion propagator (two-point function)

$$
\begin{aligned}
\frac{\delta^{2} W[\bar{I}, I, J]}{\delta \bar{I}_{a}(x) \delta I_{b}(y)} & =S_{a b}(x, y)-\int d^{4} z \bar{I}_{c}(z) \frac{\delta S_{c b}(z, y)}{\delta \bar{I}_{a}(x)} \\
& =S_{a b}(x, y)+\int d^{4} z \frac{\delta S_{a c}(x, z)}{\delta I_{b}(y)} I_{c}(z),
\end{aligned}
$$

where the functional derivatives $\delta S / \delta \bar{I}$ and $\delta S / \delta I$ can be calculated using Eq. (3.25). Combined with Eq. (3.24), we find that the $\chi$-field is the full fermion propagator if we switch off the external sources. On the vacuum, all external sources should be taken to zero, and therefore Eq. (3.25) gives

$$
S_{a c}^{-1}\left(x, x^{\prime}\right)-\left(i \not \partial_{x}\right)_{a c} \delta^{4}\left(x-x^{\prime}\right)=-i e^{2} D_{\mu \nu}\left(x, x^{\prime}\right) \gamma_{a d}^{\mu} S^{d b}\left(x, x^{\prime}\right) \gamma_{b c}^{\nu}
$$

which is just the quenched ladder approximation for the Schwinger-Dyson equation for the full fermion propagator. By differentiating the generating functional twice with respect to the source $J$ and imposing the stationary condition for the effective action, we can get the Schwinger-Dyson for the photon field.

For the four-point function, we use Eq. (3.26) and (3.27) to relate the four-point function to derivatives of the function $S$. After putting the sources equal to zero, and defining

$$
G^{a c b d}\left(x, x^{\prime}, y, y^{\prime}\right)=\left.\frac{\delta S_{a c}\left(x, x^{\prime}\right)}{\delta \bar{I}_{b}(y) \delta I_{d}\left(y^{\prime}\right)}\right|_{J=0, I=\bar{I}=0}
$$

we find for the full four-point function

$$
\begin{aligned}
\mathcal{G}^{a c b d}\left(x, x^{\prime}, y, y^{\prime}\right) & \left.\equiv \frac{\delta^{4} W[\bar{I}, I, J]}{\delta \bar{I}_{a}(x) \delta I_{c}\left(x^{\prime}\right) \delta \bar{I}_{b}(y) \delta I_{d}\left(y^{\prime}\right)}\right|_{J=0, I=\bar{I}=0} \\
& =G^{a c b d}\left(x, x^{\prime}, y, y^{\prime}\right)-G^{b c a d}\left(y, x^{\prime}, x, y^{\prime}\right) \\
& =G^{a c b d}\left(x, x^{\prime}, y, y^{\prime}\right)-G^{a d b c}\left(x, y^{\prime}, y, x^{\prime}\right)
\end{aligned}
$$

We have written down all possible expressions, which we can use to solve the four-point functions. Note that the four-point function $\mathcal{G}$ is written as the sum of the $s$ - and $t$-channel components $G$.

Using Eq. (3.25) we find for the $s$-channel 


$$
\begin{aligned}
& \int d^{4} y d^{4} y^{\prime} S_{a b}^{-1}(x, y) G^{b d a^{\prime} c^{\prime}}\left(y, y^{\prime}, x_{1}, x_{1}^{\prime}\right) S_{d c}^{-1}\left(y^{\prime}, x^{\prime}\right)= \\
& \gamma_{a d}^{\mu}\left[i G^{d b a^{\prime} c^{\prime}}\left(x, x^{\prime}, x_{1}, x_{1}^{\prime}\right)+S_{d c^{\prime}}\left(x, x_{1}^{\prime}\right) S_{a^{\prime} b}\left(x_{1}, x^{\prime}\right)\right] e^{2} D_{\mu \nu}\left(x, x^{\prime}\right) \gamma_{b c}^{\nu}
\end{aligned}
$$

This is nothing but the (inhomogeneous) Bethe-Salpeter equation. For the $S+P$ and $T$ type four-point functions in the massless-fermion phase (or more general: for all nonperturbative four-point functions), the second term on the right hand side of Eq. (3.31) vanishes, so we arrive at following equation, namely

$$
\begin{aligned}
& G^{a b c d}\left(x_{1}, x_{2}, x_{3}, x_{4}\right)= \\
& -i e^{2} \int d^{4} y d^{4} y^{\prime} S_{a d^{\prime}}\left(x_{1}, y\right) \gamma_{d^{\prime} a^{\prime}}^{\mu} G^{a^{\prime} b^{\prime} c d}\left(y, y^{\prime}, x_{3}, x_{4}\right) \gamma_{b^{\prime} c^{\prime}}^{\nu} S_{c^{\prime} b}\left(y^{\prime}, x_{2}\right) D_{\mu \nu}\left(y, y^{\prime}\right) .
\end{aligned}
$$

This equation is the $s$-channel equation for the nonperturbative four-fermion functions in the quenched ladder approximation. For the $t$-channel equation, we can get a similar equation in exactly the same way. This $t$-channel equation however is completely equivalent to the $s$-channel equation, and it can also be obtained by applying a Fierz transformation to the $s$-channel equation.

Next we make a Fourier transformation to momentum space. The equation for the $s$-channel four-point function becomes

$$
\begin{aligned}
& G^{a b c d}\left(p_{1}, p_{2}, p_{3}, p_{4}\right)= \\
& i e^{2} \int \frac{d^{4} q}{(2 \pi)^{4}} S_{a d^{\prime}}\left(p_{1}\right) \gamma_{d^{\prime} a^{\prime}}^{\mu} G^{a^{\prime} b^{\prime} c d}\left(p_{1}+q, p_{2}-q, p_{3}, p_{4}\right) \gamma_{b^{\prime} c^{\prime}}^{\nu} S_{c^{\prime} b}\left(-p_{2}\right) D_{\mu \nu}(q),
\end{aligned}
$$

where $D_{\mu \nu}(q)$ is the bare photon propagator in momentum space

$$
D_{\mu \nu}(q)=\frac{-1}{q^{2}}\left[g_{\mu \nu}-(1+a) \frac{q_{\mu} q_{\nu}}{q^{2}}\right] \text {. }
$$

As mentioned before, the $t$-channel equation is completely equivalent to this $s$-channel equation. For the complete nonperturbative four-point functions in momentum space, $\mathcal{G}\left(p_{1}, p_{2}, p_{3}, p_{4}\right)$, we thus find

$$
\begin{aligned}
\mathcal{G}^{a b c d}\left(p_{1}, p_{2}, p_{3}, p_{4}\right) & =G^{a b c d}\left(p_{1}, p_{2}, p_{3}, p_{4}\right)-G^{c b a d}\left(p_{3}, p_{2}, p_{1}, p_{4}\right) \\
& =G^{a b c d}\left(p_{1}, p_{2}, p_{3}, p_{4}\right)-G^{a d c b}\left(p_{1}, p_{4}, p_{3}, p_{2}\right)
\end{aligned}
$$

So finally we have the leading order equations for the nonperturbative four-fermion functions, which is shown in diagrams Fig. 1. For the perturbative four-fermion functions ones we have to add the tree contribution coming from the second term in the right hand side of Eq.(3.31).

\section{CALCULATION OF THE FOUR-POINT FUNCTIONS}

One can show that the perturbative four-fermion functions do not contribute at all to the nonperturbative ones, so the nonperturbative four-point functions form a closed set of equations, at least below the critical coupling for dynamical mass generation. Furthermore, since we assume that all the other types of nonperturbative four-point functions (e.g. the 
ones which break parity or Lorentz invariance) remain zero, and we only have to deal with the $S+P$ and $T$ type four-point functions

$$
\begin{aligned}
\mathcal{G}_{S+P}\left(x_{1}, x_{2}, x_{3}, x_{4}\right)= & \left\langle 0\left|\mathbf{T} \bar{\psi}\left(x_{1}\right) \psi\left(x_{2}\right) \bar{\psi}\left(x_{3}\right) \psi\left(x_{4}\right)\right| 0\right\rangle \\
& +\left\langle 0\left|\mathbf{T} \bar{\psi}\left(x_{1}\right) \gamma_{5} \psi\left(x_{2}\right) \bar{\psi}\left(x_{3}\right) \gamma_{5} \psi\left(x_{4}\right)\right| 0\right\rangle, \\
\mathcal{G}_{T}\left(x_{1}, x_{2}, x_{3}, x_{4}\right)= & \left\langle 0\left|\mathbf{T} \bar{\psi}\left(x_{1}\right) \sigma_{\mu \nu} \psi\left(x_{2}\right) \bar{\psi}\left(x_{3}\right) \sigma^{\mu \nu} \psi\left(x_{4}\right)\right| 0\right\rangle .
\end{aligned}
$$

Using the general nonperturbative equation for the four-fermion function derived in the previous section, we can project out the equations for these $S+P$ and $T$ type four-point functions, which form closed set of linear integral equations.

The four-fermion functions $\mathcal{G}_{\mathcal{S}+\mathcal{P}}$ and $\mathcal{G}_{\mathcal{T}}$ are related to the functions $G_{S+P}$ and $G_{T}$ by

$$
\begin{aligned}
\mathcal{G}_{S+P}\left(p_{1}, p_{2}, p_{3}, p_{4}\right) & =G_{S+P}\left(p_{1}, p_{2}, p_{3}, p_{4}\right)-\frac{1}{2} G_{S+P}\left(p_{3}, p_{2}, p_{1}, p_{4}\right)-3 G_{T}\left(p_{3}, p_{2}, p_{1}, p_{4}\right) \\
& =G_{S+P}\left(p_{1}, p_{2}, p_{3}, p_{4}\right)-\frac{1}{2} G_{S+P}\left(p_{1}, p_{4}, p_{3}, p_{2}\right)-3 G_{T}\left(p_{1}, p_{4}, p_{3}, p_{2}\right) \\
\mathcal{G}_{T}\left(p_{1}, p_{2}, p_{3}, p_{4}\right) & =G_{T}\left(p_{1}, p_{2}, p_{3}, p_{4}\right)-\frac{1}{4} G_{S+P}\left(p_{3}, p_{2}, p_{1}, p_{4}\right)+\frac{1}{4} G_{T}\left(p_{3}, p_{2}, p_{1}, p_{4}\right) \\
& =G_{T}\left(p_{1}, p_{2}, p_{3}, p_{4}\right)-\frac{1}{4} G_{S+P}\left(p_{1}, p_{4}, p_{3}, p_{2}\right)+\frac{1}{4} G_{T}\left(p_{1}, p_{4}, p_{3}, p_{2}\right)
\end{aligned}
$$

After the projection and reduction of the $\gamma$-algebra, we get for the $s$-channel $S+P$ and $T$ type four-fermion functions

$$
\begin{aligned}
G_{S+P}\left(p_{1}, p_{2}, p_{3}, p_{4}\right) & =i e^{2} \frac{p_{1} \cdot p_{2}}{p_{1}^{2} p_{2}^{2}} \int \frac{d^{4} q}{(2 \pi)^{4}} D_{\mu}^{\mu}(q) G_{S+P}\left(p_{1}-q, p_{2}+q, p_{3}, p_{4}\right) \\
G_{T}\left(p_{1}, p_{2}, p_{3}, p_{4}\right) & =i e^{2} \frac{4 p_{1}^{\mu} p_{2}^{\nu}-p_{1} \cdot p_{2} g^{\mu \nu}}{3 p_{1}^{2} p_{2}^{2}} \int \frac{d^{4} q}{(2 \pi)^{4}} D_{\mu \nu}(q) G_{T}\left(p_{1}-q, p_{2}+q, p_{3}, p_{4}\right)
\end{aligned}
$$

where $D_{\mu}^{\mu}(q)$ is the bare photon propagator. Remember that in $G_{S+P}\left(p_{1}, p_{2}, p_{3}, p_{4}\right)$ and $G_{T}\left(p_{1}, p_{2}, p_{3}, p_{4}\right)$, momentum conservation condition $p_{1}+p_{2}+p_{3}+p_{4}=0$ should be satisfied. Since the $t$-channel equations follow from these $s$-channel equations, it is enough to analyze these two $s$-channel equations, at least for the question whether or not there is a critical coupling.

We also have to choose gauge. If we take the Feynman gauge, then the equation Eq. (4.8) gives immediately $G_{T}\left(p_{1}, p_{2}, p_{3}, p_{4}\right)=0$, whereas the equation for $G_{S+P}$, Eq. (4.7), leads automatically to $G_{S+P}\left(p_{1}, p_{2}, p_{3}, p_{4}\right)=0$ in the Yennie gauge. These results are obviously artifacts of the specific gauges, and one should choose the "correct" gauge in order to get reasonable results. It is known that the quenched ladder approximation for the fermion Schwinger-Dyson equation, which also follows from our effective action, is most reliable in the Landau gauge, $a=0$. Therefore, we analyze the equations for these four-fermion functions in the Landau gauge, and use the photon propagator

$$
D_{\mu \nu}(q)=\frac{-1}{q^{2}}\left(g_{\mu \nu}-\frac{q_{\mu} q_{\nu}}{q^{2}}\right) .
$$




\section{A. $G_{S+P}$}

We start by looking at the equation for $G_{S+P}$, Eq. (4.7), which becomes in Euclidean space, after a Wick rotation,

$$
G_{S+P}\left(p_{1}, p_{2}, p_{3}, p_{4}\right)=-3 e^{2} \frac{p_{1} \cdot p_{2}}{p_{1}^{2} p_{2}^{2}} \int \frac{d^{4} q}{(2 \pi)^{4}} \frac{1}{q^{2}} G_{S+P}\left(p_{1}-q, p_{2}+q, p_{3}, p_{4}\right) .
$$

We redefine

$$
\check{G}_{S+P}\left(p_{1}, p_{2}, p_{3}, p_{4}\right) \equiv G_{S+P}\left(p_{1}, p_{2}-p_{1}, p_{3}, p_{4}\right)
$$

which allows us to rewrite this equation into

$$
\check{G}_{S+P}\left(p_{1}, p_{2}, p_{3}, p_{4}\right)=-3 e^{2} \frac{p_{1} \cdot\left(p_{2}-p_{1}\right)}{p_{1}^{2}\left(p_{2}-p_{1}\right)^{2}} \int \frac{d^{4} q}{(2 \pi)^{4}} \frac{1}{\left(q-p_{1}\right)^{2}} \check{G}_{S+P}\left(q, p_{2}, p_{3}, p_{4}\right) .
$$

In order to solve this equation, we first expand $\hat{G}_{S+P}\left(p_{1}, p_{2}, p_{3}, p_{4}\right)$ in terms of $\hat{p}_{2}^{\mu}$

$$
\check{G}_{S+P}\left(p_{1}, p_{2}, p_{3}, p_{4}\right)=\sum_{n=0}^{\infty}\left(\hat{p}_{2}\right)_{\mu_{1}} \ldots\left(\hat{p}_{2}\right)_{\mu_{n}} \tilde{G}_{n}^{\mu_{1} \ldots \mu_{n}}\left(p_{1}, p_{2 r}, p_{3}, p_{4}\right)
$$

where we use the notation $\hat{p}_{i}^{\mu} \equiv p_{i}^{\mu} / \sqrt{p_{i}^{2}}$ for the angular dependence, and $p_{i r} \equiv \sqrt{p_{i}^{2}}$ for the absolute value of the momenta. Note also that $\tilde{G}_{n}^{\mu_{1} \ldots \mu_{n}}\left(p_{1}, p_{2 r}, p_{3}, p_{4}\right)$ is symmetric in the indices $\mu_{1}, \ldots, \mu_{n}$. Inserting this in Eq. (4.12) shows immediately that the coefficients $\tilde{G}_{n}^{\mu_{1} \ldots \mu_{n}}\left(p_{1}, p_{2 r}, p_{3}, p_{4}\right)$ have to satisfy

$$
\begin{array}{r}
p_{1}^{2} \tilde{G}_{0}\left(p_{1}, p_{2 r}, p_{3}, p_{4}\right)=3 e^{2} \int \frac{d^{4} q}{(2 \pi)^{4}} \frac{1}{\left(q-p_{1}\right)^{2}} \tilde{G}_{0}\left(q, p_{2 r}, p_{3}, p_{4}\right), \\
-2 p_{1}^{\mu_{1}} p_{1}^{2} \tilde{G}_{0}\left(p_{1}, p_{2 r}, p_{3}, p_{4}\right)+p_{1}^{4} \tilde{G}_{1}^{\mu_{1}}\left(p_{1}, p_{2 r}, p_{3}, p_{4}\right)=3 e^{2} \int \frac{d^{4} q}{(2 \pi)^{4}} \frac{1}{\left(q-p_{1}\right)^{2}} \times \\
{\left[-p_{1}^{\mu_{1}} \tilde{G}_{0}\left(q, p_{2 r}, p_{3}, p_{4}\right)+p_{1}^{2} \tilde{G}_{1}^{\mu_{1}}\left(q, p_{2 r}, p_{3}, p_{4}\right)\right],}
\end{array}
$$

and

$$
\begin{aligned}
& {\left[\delta^{\mu_{1} \mu_{2}} p_{1}^{2} \tilde{G}_{n-2}^{\mu_{3} \ldots \mu_{n}}\left(p_{1}, p_{2 r}, p_{3}, p_{4}\right)-2 p_{1}^{\mu_{1}} p_{1}^{2} \tilde{G}_{n-1}^{\mu_{2} \ldots \mu_{n}}\left(p_{1}, p_{2 r}, p_{3}, p_{4}\right)+p_{1}^{4} \tilde{G}_{n}^{\mu_{1} \ldots \mu_{n}}\left(p_{1}, p_{2 r}, p_{3}, p_{4}\right)\right.} \\
& \left.\quad+\text { all symmetric permutations in }\left(\mu_{1} \ldots \mu_{n}\right)\right] \\
& =3 e^{2} \int \frac{d^{4} q}{(2 \pi)^{4}} \frac{1}{\left(q-p_{1}\right)^{2}}\left[-p_{1}^{\mu_{1}} \tilde{G}_{n-1}^{\mu_{2} \ldots \mu_{n}}\left(q, p_{2 r}, p_{3}, p_{4}\right)+p_{1}^{2} \tilde{G}_{n}^{\mu_{1} \ldots \mu_{n}}\left(q, p_{2 r}, p_{3}, p_{4}\right)\right. \\
& \left.\quad+\text { all symmetric permutations in }\left(\mu_{1} \ldots \mu_{n}\right)\right]
\end{aligned}
$$

with $n=2,3,4, \ldots$. The homogeneous part of above equation satisfies

$$
p_{1}^{2} \tilde{G}_{n}^{\mu_{1} \ldots \mu_{n}}\left(p_{1}, p_{2 r}, p_{3}, p_{4}\right)=3 e^{2} \int \frac{d^{4} q}{(2 \pi)^{4}} \frac{1}{\left(q-p_{1}\right)^{2}} \tilde{G}_{n}^{\mu_{1} \ldots \mu_{n}}\left(q, p_{2 r}, p_{3}, p_{4}\right) .
$$


In order to prove that the only solution of the equations Eqs. (4.14)-(4.16) is the trivial solution, it is enough to prove that the only solution of the homogeneous equation Eq. (4.17) is the trivial solution $\tilde{G}_{n}^{\mu_{1} \ldots \mu_{n}}\left(p_{1}, p_{2 r}, p_{3}, p_{4}\right)=0$, for all $n=0,1,2, \ldots$. Note that only a solution which is symmetric in the Lorentz indices $\mu_{1} \ldots \mu_{n}$ will contribute to the four-point function $\check{G}_{S+P}$; from Eq. (4.13) it follows immediately that any nonsymmetric part does not contribute.

The angular integration in Eq. (4.17) can be performed analytically, using an expansion in a kind of generalized Chebyshev polynomials

$$
\begin{aligned}
\hat{U}_{2 n}^{\mu_{1} \ldots \mu_{2 n}}(\hat{q})= & \sum_{k=0}^{n} \frac{(-1)^{k} 2^{2 n-2 k}(2 n-k) !}{k !(2 n-2 k) !(2 n) !}\left[\hat{q}^{\mu_{1}} \ldots \hat{q}^{\mu_{2 n-2 k}} \delta^{\mu_{2 n-2 k+1} \mu_{2 n-2 k+2}} \ldots \delta^{\mu_{2 n-1} \mu_{2 n}}\right. \\
& \left.+ \text { all symmetric permutations in }\left(\mu_{1} \ldots \mu_{2 n}\right)\right], \\
\hat{U}_{2 n+1}^{\mu_{1} \ldots \mu_{2 n+1}}(\hat{q})= & \sum_{k=0}^{n} \frac{(-1)^{k} 2^{2 n+1-2 k}(2 n+1-k) !}{k !(2 n+1-2 k) !(2 n+1) !}\left[\hat{q}^{\mu_{1}} \ldots \hat{q}^{\mu_{2 n+1-2 k}} \delta^{\mu_{2 n+2-2 k} \mu_{2 n+3-2 k}} \ldots \delta^{\mu_{2 n} \mu_{2 n+1}}\right. \\
& \left.+ \text { all symmetric permutations in }\left(\mu_{1} \ldots \mu_{2 n+1}\right)\right],
\end{aligned}
$$

where $\hat{q}^{\mu}=q^{\mu} / \sqrt{q^{2}}$. These polynomials satisfy the identity

$$
\int d \Omega_{q} \frac{\hat{U}_{n}^{\mu_{1} \ldots \mu_{n}}(\hat{q})}{\left(q-p_{1}\right)^{2}}=\hat{U}_{n}^{\mu_{1} \ldots \mu_{n}}\left(\hat{p}_{1}\right) \frac{1}{(n+1) p_{1 r} q_{r}}\left[\frac{\min \left(p_{1 r}, q_{r}\right)}{\max \left(p_{1 r}, q_{r}\right)}\right]^{n+1},
$$

which is shown in Appendix A, where we have given more details on these polynomials. Assuming that our four-point function is smooth enough (or more precisely, has a Taylorexpansion), we expand the unknown functions $\tilde{G}_{n}^{\mu_{1} \ldots \mu_{n}}\left(p_{1}, p_{2 r}, p_{3}, p_{4}\right)$ in terms of these generalized Chebyshev polynomials

$$
\tilde{G}_{n}^{\mu_{1} \ldots \mu_{n}}\left(p_{1}, p_{2 r}, p_{3}, p_{4}\right)=\sum_{l=0}^{\infty} \widetilde{G_{l}^{n}} \mu_{\nu_{1} \ldots \nu_{l}}^{\mu_{1} \ldots \mu_{n}}\left(p_{1 r}, p_{2 r}, p_{3}, p_{4}\right) \hat{U}_{l}^{\nu_{1} \ldots \nu_{l}}\left(\hat{p}_{1}\right) .
$$

After this decomposition, which allows us to perform the angular integration, Eq. (4.17) has to hold for each function $\widetilde{G}_{l}^{n}$ separately, which leads to an equation for each coefficient

$$
\begin{aligned}
p_{1}^{2}{\widetilde{G_{l \nu_{1} \ldots \nu_{l}}^{n}}}^{\mu_{1} \ldots \mu_{n}}\left(p_{1 r}, p_{2 r}, p_{3}, p_{4}\right)= & \frac{3 e^{2}}{16 \pi^{2}}\left\{\int_{\epsilon^{2}}^{p_{1}^{2}} d q^{2}{\widetilde{G_{l}^{n}}}^{\mu_{1} \ldots \mu_{n}}\left(q_{r}, p_{2 r}, p_{3}, p_{4}\right)\right. \\
& \left.+\int_{p_{1}^{2}}^{\Lambda^{2}} d q^{2}{\widetilde{G_{l}^{n}}}^{\mu_{1} \ldots \mu_{n}}\left(q_{r}, p_{2 r}, p_{3}, p_{4}\right) \frac{p_{1}^{2}}{q^{2}}\right\} .
\end{aligned}
$$

For convenience we will drop all irrelevant indices and the spectator momenta in the discussion of this equation, defining $g(p)$ as a scalar function of the absolute value of $p_{1}$

\footnotetext{
${ }^{2}$ This expansion can always be made in a unique way, and can be thought of as an expansion in $\hat{p}_{1}^{\mu_{1}} \ldots \hat{p}_{1}^{\mu_{m}}$ first, followed by an expansion of $\hat{p}_{1}^{\mu_{1}} \ldots \hat{p}_{1}^{\mu_{m}}$ in terms of $\hat{U}_{l}^{\nu_{1} \ldots \nu_{l}}\left(\hat{p}_{1}\right)$ using Eqs. (A39) and (A40), see the appendix.
} 


$$
\widetilde{G_{l}^{n}{ }_{\nu_{1} \ldots \nu_{l}}}{ }^{\mu_{1} \ldots \mu_{n}}\left(p_{1 r}, p_{2 r}, p_{3}, p_{4}\right) \equiv g(p),
$$

so the equation we have to consider becomes

$$
p^{2} g(p)=\frac{3 e^{2}}{16 \pi^{2}}\left\{\int_{\epsilon^{2}}^{p^{2}} d q^{2} g(q)+\int_{p^{2}}^{\Lambda^{2}} d q^{2} \frac{p^{2}}{q^{2}} g(q)\right\} .
$$

This integral equation is exactly the same as the equation for the dynamical mass function in quenched ladder QED, which has been studied extensively [14 [16]. It can be transformed into the following differential equation

$$
\frac{d}{d p^{2}} \frac{d}{d p^{2}} p^{2} g(p)=-\frac{3 e^{2}}{16 \pi^{2}} \frac{g(p)}{p^{2}} .
$$

There are two independent solutions for the above differential equation, say $\left(p^{2}\right)^{b_{i}}$ with $b_{i}$ satisfying

$$
b_{i}=\frac{1}{2}\left[-1 \pm \sqrt{1-\frac{3 \alpha}{\pi}}\right] .
$$

From this equation, it follows immediately that there is a critical coupling $\alpha_{c}=\pi / 3$, below which both $b_{i}$ are real, and above which they are complex.

So the general solution is

$$
g(p)=\sum_{i=1,2} c_{i} p^{2 b_{i}},
$$

in terms of the two independent solutions of the differential equation, with $c_{i}$ some arbitrary functions, depending on the spectator momenta $p_{2 r}, p_{3}$, and $p_{4}$ in the original equation Eq. (4.24), which needs to be determined. This general solution is constrained by the integral equation, and therefore, we substitute this solution back in the original integral equation, which gives us

$$
\sum_{i=1,2} c_{i}\left[-\frac{\epsilon^{2 b_{i}+2}}{\left(b_{i}+1\right)}+p^{2} \frac{\Lambda^{2 b_{i}}}{b_{i}}\right] \cdot=0
$$

This boundary condition can only be satisfied above the critical coupling, if the exponents $b_{i}$ are complex; below the critical coupling, both $b_{i}$ are real numbers, and it is impossible to satisfy this boundary condition. Assuming there is a nontrivial solution, Eq. (4.28) is equivalent to the set of equations

$$
\begin{aligned}
& \frac{c_{1}}{c_{2}}=-\frac{b_{1}+1}{b_{2}+1} \epsilon^{2\left(b_{2}-b_{1}\right)}, \\
& \frac{c_{1}}{c_{2}}=-\frac{b_{1}}{b_{2}} \Lambda^{2\left(b_{2}-b_{1}\right)} .
\end{aligned}
$$

Below the critical coupling, this set of equations only has a solution if $\epsilon>\Lambda$, which means the infrared cutoff bigger than the ultraviolet cutoff. This is obviously not a physical solution, 
and therefore the only solution of Eq. (4.25) is the trivial solution. That means that the only solution of the integral equation Eq (4.22) is the trivial solution

$$
{\widetilde{G_{l}^{n} \nu_{1} \ldots \nu_{l}}}^{\mu_{1} \ldots \mu_{n}}\left(p_{1 r}, p_{2 r}, p_{3}, p_{4}\right)=0
$$

Now going back to the original equation for the $S+P$ type four-fermion function, using the decomposition Eqs. (4.21) and (4.13), and definition Eq. (4.11), we find that only the trivial solution

$$
G_{S+P}\left(p_{1}, p_{2}, p_{3}, p_{4}\right)=0
$$

is allowed.

Above the critical coupling, $\alpha>\pi / 3$, both $b_{i}$ have a nonzero imaginary part, which implies oscillating solutions. Instead of using the complex exponents $b_{i}$, we write the general solution of the differential equation Eq. (4.25) as

$$
g(p)=\frac{c_{1}}{p} \sin \left(\sqrt{\frac{3 \alpha}{\pi}-1} \ln \frac{p}{\Lambda}\right)+\frac{c_{2}}{p} \cos \left(\sqrt{\frac{3 \alpha}{\pi}-1} \ln \frac{p}{\Lambda}\right),
$$

where we have used the ultraviolet cut-off $\Lambda$ to set the mass scale. Substituting this solution back in original integral equation, we finally find a condition for $\Lambda / \epsilon$

$$
\begin{aligned}
\sqrt{\frac{3 \alpha}{\pi}-1} \ln \left(\frac{\Lambda}{\epsilon}\right) & =\left(2 n+\frac{1}{2}\right) \pi+\delta \quad n \text { integer } \\
\tan \delta & =\sqrt{\frac{3 \alpha}{\pi}-1} .
\end{aligned}
$$

Now one can always construct nontrivial solutions, depending on this ratio $\epsilon / \Lambda$. We assume that $\epsilon$, the infrared cutoff, is related to the energy scale of the dynamically generated condensate through nonlinear effects, just as in the linearized quenched ladder approximation for the fermion Schwinger-Dyson equation, where the infrared cut-off sets the scale for the infrared mass function, $\epsilon \simeq m(0)$. Thus we allow the infrared cut-off to be a function of the ultraviolet cut-off, and in general, the lowest value of $n$ for which $\epsilon<\Lambda$ will correspond to the ground state solution; higher values of $n$ correspond to oscillating solutions, i.e. excited states with higher energy.

So there is a critical coupling $\alpha_{c}=\pi / 3$, above which one can find a nontrivial solution for the homogeneous equation, which is also the zeroth-order solution for $G\left(p_{1}, p_{2}, p_{3}, p_{4}\right)$ if one expand it in terms of $p_{2}^{\mu}$. The only special point is that the ratio $\epsilon / \Lambda$ must satisfy some constraint. In the limit that the coupling goes towards the critical coupling, $\alpha \downarrow \alpha_{c}$, this ratio goes to infinity, and we can remove the ultraviolet and infrared cut-offs.

Note that this critical coupling coincides with the critical coupling for dynamical mass generation for the fermions in the quenched ladder approximation. This is no surprise, since we noted already that we arrived at the same integral equation as that for the mass function, $\mathrm{Eq}$ (4.24). Looking back at the original equation for $G_{S+P}$, Eq. (4.10), we can understand this, because for $p_{1}=p_{2}$ this equation is exactly the same as the linearized quenched ladder approximation for the fermion propagator. 
B. $G_{T}$

Now we calculate $G_{T}$, Eq. (4.8), which becomes in Euclidean space

$$
G_{T}\left(p_{1}, p_{2}, p_{3}, p_{4}\right)=e^{2} \frac{4 p_{1}^{\mu} p_{2}^{\nu}-p_{1} \cdot p_{2} \delta^{\mu \nu}}{3 p_{1}^{2} p_{2}^{2}} \int \frac{d^{4} q}{(2 \pi)^{4}} \frac{q_{\mu} q_{\nu}}{q^{4}} G_{T}\left(p_{1}-q, p_{2}+q, p_{3}, p_{4}\right) .
$$

We can solve this equation in the same way as the $S+P$ type equation, redefining

$$
\check{G}_{T}\left(p_{1}, p_{2}, p_{3}, p_{4}\right) \equiv G_{T}\left(p_{1}, p_{2}-p_{1}, p_{3}, p_{4}\right)
$$

which allows us to rewrite this equation into

$$
\begin{aligned}
\check{G}_{T}\left(p_{1}, p_{2}, p_{3}, p_{4}\right)= & e^{2} \frac{4 p_{1}^{\mu}\left(p_{2}-p_{1}\right)^{\nu}-p_{1} \cdot\left(p_{2}-p_{1}\right) \delta^{\mu \nu}}{3 p_{1}^{2}\left(p_{2}-p_{1}\right)^{2}} \times \\
& \int \frac{d^{4} q}{(2 \pi)^{4}} \frac{\left(q-p_{1}\right)_{\mu}\left(q-p_{1}\right)_{\nu}}{\left(q-p_{1}\right)^{4}} \check{G}_{T}\left(q, p_{2}, p_{3}, p_{4}\right) .
\end{aligned}
$$

Similarly, we expand $\hat{G}_{T}\left(p_{1}, p_{2}, p_{3}, p_{4}\right)$ in terms of $\hat{p}_{2}^{\mu}$

$$
\check{G}_{T}\left(p_{1}, p_{2}, p_{3}, p_{4}\right)=\sum_{n=0}^{\infty}\left(\hat{p}_{2}\right)_{\mu_{1}} \ldots\left(\hat{p}_{2}\right)_{\mu_{n}} \tilde{G}_{n}^{\mu_{1} \ldots \mu_{n}}\left(p_{1}, p_{2 r}, p_{3}, p_{4}\right)
$$

Inserting this in Eq. (4.38) leads to a set of equations for the functions $\tilde{G}_{n}^{\mu_{1} \ldots \mu_{n}}\left(p_{1}, p_{2 r}, p_{3}, p_{4}\right)$, and in order to show that the only solution for Eq. (4.38) is the trivial solution, it is enough to prove that the only solution of the equation

$$
\begin{aligned}
& p_{1}^{4} \tilde{G}_{n}^{\mu_{1} \ldots \mu_{n}}\left(p_{1}, p_{2 r}, p_{3}, p_{4}\right)= \\
& =e^{2}\left(-4 p_{1}^{\mu} p_{1}^{\nu}+\delta^{\mu \nu} p_{1}^{2}\right) \int \frac{d^{4} q}{(2 \pi)^{4}} \frac{\left(q_{\mu}-p_{1 \mu}\right)\left(q_{\nu}-p_{1 \nu}\right)}{\left(q-p_{1}\right)^{4}} \tilde{G}_{n}^{\mu_{1} \ldots \mu_{n}}\left(q, p_{2 r}, p_{3}, p_{4}\right)
\end{aligned}
$$

is the trivial solution $\tilde{G}_{n}^{\mu_{1} \ldots \mu_{n}}\left(p_{1}, p_{2 r}, p_{3}, p_{4}\right)=0$, for all $n=0,1,2, \ldots$, just as before.

The generalized Chebyshev polynomials satisfy the identity

$$
\begin{aligned}
& \int d \Omega_{q} \frac{\left(q_{\mu}-p_{1 \mu}\right)\left(q_{\nu}-p_{1 \nu}\right)\left(4 p_{1}^{\mu} p_{1}^{\nu}-\delta^{\mu \nu} p_{1}^{2}\right)}{\left(q-p_{1}\right)^{4}} \hat{U}_{n}^{\mu_{1} \ldots \mu_{n}}(\hat{q})= \\
& =\delta_{n 0}+\hat{U}_{n}^{\mu_{1} \ldots \mu_{n}}\left(\hat{p}_{1}\right) \frac{1}{n+1}\left[-q^{2}-\left(p_{1}^{2}-q^{2}\right) p_{1 r} \frac{d}{d p_{1 r}}\right] \frac{1}{p_{1 r} q_{r}}\left[\frac{\min \left(p_{1 r}, q_{r}\right)}{\max \left(p_{1 r}, q_{r}\right)}\right]^{n+1}
\end{aligned}
$$

so if we expand the unknown functions $\tilde{G}_{n}^{\mu_{1} \ldots \mu_{n}}\left(p_{1}, p_{2 r}, p_{3}, p_{4}\right)$ in terms of these polynomials

$$
\tilde{G}_{n}^{\mu_{1} \ldots \mu_{n}}\left(p_{1}, p_{2 r}, p_{3}, p_{4}\right)=\sum_{l=0}^{\infty}{\widetilde{G_{l}^{n}}}^{\mu_{1} \ldots \mu_{n}}{ }^{\mu_{1}}\left(p_{1 r}, p_{2 r}, p_{3}, p_{4}\right) \hat{U}_{l}^{\nu_{1} \ldots \nu_{l}}\left(\hat{p}_{1}\right)
$$

we can perform the angular integrals in Eq. (4.40) analytically, see Appendix A. This gives an equation for each of the coefficients separately 


$$
\begin{aligned}
p_{1}^{4} & \widetilde{G_{l}^{n}} \mu_{\nu_{1} \ldots \nu_{l}}^{\mu_{1} \ldots \mu_{n}}\left(p_{1 r}, p_{2 r}, p_{3}, p_{4}\right)= \\
= & -\frac{e^{2}}{48 \pi^{2}}\left\{\int_{\epsilon^{2}}^{p_{1}^{2}} d q^{2}{\widetilde{G_{l}^{n}}}_{l \nu_{1} \ldots \nu_{l}}^{\mu_{1} \ldots \mu_{n}}\left(q_{r}, p_{2 r}, p_{3}, p_{4}\right) q^{2}\left[\delta_{l 0}+\left(\frac{l+2}{l+1}-\frac{l+3}{l+1} \frac{q^{2}}{p_{1}^{2}}\right)\left(\frac{q_{r}}{p_{1 r}}\right)^{l}\right]\right. \\
& \left.+\int_{p_{1}^{2}}^{\Lambda^{2}} d q^{2} \widetilde{G_{l}^{n}}{\widetilde{\nu_{1} \ldots \nu_{l}}}^{\mu_{1} \ldots \mu_{n}}\left(q_{r}, p_{2 r}, p_{3}, p_{4}\right) q^{2}\left[\delta_{l 0}+\left(-\frac{l}{l+1} \frac{p_{1}^{2}}{q^{2}}+\frac{l-1}{l+1}\right)\left(\frac{p_{1 r}}{q_{r}}\right)^{l}\right]\right\} .
\end{aligned}
$$

Again, for convenience we will drop all irrelevant indices and the spectator momenta in the discussion of this equation, defining $g(p)$ as a scalar function of the absolute value of $p_{1}$

$$
{\widetilde{G_{l}^{n}}}_{\mu_{1} \ldots \nu_{l}}^{\mu_{1} \ldots \mu_{n}}\left(p_{1 r}, p_{2 r}, p_{3}, p_{4}\right) \equiv g\left(p_{1}\right),
$$

so the equation we have to consider becomes

$$
\begin{aligned}
p^{4} g(p)= & -\frac{e^{2}}{48 \pi^{2}}\left\{\int_{\epsilon^{2}}^{p^{2}} d q^{2} q^{2} g(q)\left[\delta_{l 0}+\left(\frac{l+2}{l+1}-\frac{l+3}{l+1} \frac{q^{2}}{p^{2}}\right)\left(\frac{q}{p}\right)^{l}\right]\right. \\
& \left.+\int_{p^{2}}^{\Lambda^{2}} d q^{2} q^{2} g(q)\left[\delta_{l 0}+\left(-\frac{l}{l+1} \frac{p^{2}}{q^{2}}+\frac{l-1}{l+1}\right)\left(\frac{p}{q}\right)^{l}\right]\right\} .
\end{aligned}
$$

Since the $l=0$ and $l \neq 0$ cases are different, we discuss them separately.

For $l=0$, the integral equation can be transformed into the following differential equation

$$
\frac{d}{d p^{2}} \frac{d}{d p^{2}} p^{6} g(p)=-\frac{e^{2}}{16 \pi^{2}} p^{2} g(p) .
$$

There are two independent solutions for the above differential equation, say $\left(p^{2}\right)^{b-2}$ with $b$ satisfying

$$
b=\frac{1}{2}\left[-1 \pm \sqrt{1-\frac{\alpha}{\pi}}\right] .
$$

This means that the general solution of the differential equation can be written as

$$
g(p)=\sum_{i=1,2} c_{i} p^{2 b_{i}-2},
$$

with $c_{i}$ some arbitrary functions, depending on the spectator momenta $p_{2 r}, p_{3}$, and $p_{4}$ in the original equation. We substitute this solution back in the original integral equation in order to get the correct boundary conditions, which gives us

$$
\sum_{i=1,2} c_{i}\left[\frac{\epsilon^{2 b_{i}}}{b_{i}-1}-\frac{\epsilon^{2 b_{i}+2}}{p^{2} b_{i}}\right]=0 .
$$

The critical coupling for the nontrivial solution is $\alpha_{c}^{0}=\pi$, as can be seen from Eq. (4.47), and just as for the $S+P$ type equation, below the critical coupling this constraint allows only the trivial solution

$$
g(p)=0 .
$$


Above this critical coupling, were the roots $b_{i}$ become complex, we can construct a nontrivial solution similar as before.

For $l \neq 0$, the integral equation can be transformed into the following differential equation

$$
\begin{aligned}
& \frac{d}{d p^{2}} \frac{d}{d p^{2}}\left(p^{2}\right)^{l+3} \frac{d}{d p^{2}} \frac{d}{d p^{2}}\left(p^{2}\right)^{-\frac{l}{2}+2} g(p)= \\
& =\frac{e^{2}}{48 \pi^{2}}\left[\frac{d}{d p^{2}} \frac{d}{d p^{2}}\left(p^{2}\right)^{\frac{l}{2}+3} g(p)-3(l+2) \frac{d}{d p^{2}}\left(p^{2}\right)^{\frac{l}{2}+2} g(p)+l(l+2)\left(p^{2}\right)^{\frac{l}{2}+1} g(p)\right] .
\end{aligned}
$$

Now there are four independent solutions for the above differential equation, say $\left(p^{2}\right)^{b^{\prime}-2-\frac{l}{2}}$ with $b^{\prime}$ satisfying the equation

$$
f_{l}^{\alpha}\left(b^{\prime}\right) \equiv b^{\prime}\left(b^{\prime}+1\right)\left(b^{\prime}-l\right)\left(b^{\prime}-l-1\right)+\frac{\alpha}{12 \pi}\left[3 b^{\prime}\left(b^{\prime}-l-1\right)+l(l+2)\right]=0 .
$$

We can calculate some special values $f_{l}^{\alpha}\left(b^{\prime}\right)$, to see where the roots are located, and find for small values of the coupling

$$
\begin{aligned}
f_{l}^{\alpha}(l+1) & =\frac{\alpha}{12 \pi} l(l+2)>0 \\
f_{l}^{\alpha}\left(l+\frac{1}{2}\right) & =-\frac{1}{4}\left[l^{2}+2 l+\frac{3}{4}-\frac{\alpha}{3 \pi}\left(l^{2}+\frac{l}{2}-\frac{3}{4}\right)\right]<0 \\
f_{l}^{\alpha}(l) & =\frac{\alpha}{12 \pi} l(l-1) \geq 0 \\
f_{l}^{\alpha}(0) & =\frac{\alpha}{12 \pi} l(l+2)>0 \\
f_{l}^{\alpha}\left(-\frac{1}{2}\right) & =-\frac{1}{4}\left[l^{2}+2 l+\frac{3}{4}-\frac{\alpha}{3 \pi}\left(l^{2}+\frac{7 l}{2}+\frac{9}{4}\right)\right]<0 \\
f_{l}^{\alpha}(-1) & =\frac{\alpha}{12 \pi}(l+2)(l+3)>0
\end{aligned}
$$

From this it follows immediately that at small values of the coupling all four roots of the equation are real: two of them are positive and the other two are negative. In principle, there might be a critical coupling $\alpha_{c}$, where two roots become degenerate; such a critical coupling satisfies the equations

$$
\begin{gathered}
f_{l}^{\alpha}\left(b^{\prime}\right)=0 \\
f_{l}^{\prime \alpha}\left(b^{\prime}\right)=0 .
\end{gathered}
$$

This critical coupling will in general depend on the parameter $l$, and from Eq. (4.53) we can see immediately that a lower bound for these critical coupling is

$$
\begin{aligned}
& \alpha_{c}^{1} \geq \frac{5}{3} \pi, \\
& \alpha_{c}^{2} \geq \frac{105}{53} \pi, \\
& \alpha_{c}^{3} \geq \frac{189}{87} \pi, \\
& \ldots
\end{aligned}
$$

A more detailed calculations shows that the critical coupling for $l=1$ is indeed $\alpha_{c}^{1}=\frac{5}{3} \pi$. 
Again, the general solution can be written as

$$
g(p)=\sum_{i=1,2,3,4} c_{i} p^{2 b_{i}^{\prime}-4-l},
$$

with $c_{i}$ depending on the spectator momenta $p_{2 r}, p_{3}$, and $p_{4}$ in the original equation Eq. (4.43), and constrained by the integral equation. Therefore, we substitute this solution back in the integral equation, which gives us 3

$$
\sum_{i=1}^{4} c_{i}\left[\frac{l+2}{l+1} \frac{\epsilon^{2 b_{i}^{\prime}}}{p^{l} b_{i}^{\prime}}+\frac{l+3}{l+1} \frac{\epsilon^{2 b_{i}^{\prime}+2}}{p^{l+2}\left(b_{i}^{\prime}+1\right)}-\frac{l}{l+1} \frac{p^{l+2} \Lambda^{2 b_{i}^{\prime}-2 l-2}}{b_{i}^{\prime}-l-1}+\frac{l-1}{l+1} \frac{p^{l} \Lambda^{2 b_{i}^{\prime}-2 l}}{b_{i}^{\prime}-l}\right]=0,
$$

which is equivalent to

$$
\begin{aligned}
\sum_{i=1}^{4} c_{i} \frac{\epsilon^{2 b_{i}^{\prime}}}{b_{i}^{\prime}} & =0, \\
\sum_{i=1}^{4} c_{i} \frac{\epsilon^{2 b_{i}^{\prime}+2}}{\left(b_{i}^{\prime}+1\right)} & =0, \\
\sum_{i=1}^{4} c_{i} \frac{\Lambda^{2 b_{i}^{\prime}-2 l-2}}{b_{i}^{\prime}-l-1} & =0, \\
\sum_{i=1}^{4} c_{i} \frac{l-1}{b_{i}^{\prime}-l} \frac{\Lambda^{2 b_{i}^{\prime}-2 l}}{l+1} & =0 .
\end{aligned}
$$

We also have the additional constraint that the infrared cut-off is smaller than the ultraviolet cut-off. This set of equations has no nontrivial solution below the critical coupling, with all the roots $b_{i}^{\prime}$ real, but above the critical coupling, some of $b_{i}^{\prime}$ develop an imaginary part which means that the solution starts oscillating. Just as in the case of $G_{S+P}$, these oscillations allow for nontrivial solutions of the integral equation, with $\epsilon<\Lambda$.

Note, that the critical coupling for $G_{T}$ depends on $l$, and all of them are bigger than that for $G_{S+P}$ which is $\frac{\pi}{3}$; the smallest one, corresponding to $l=0$ is $\alpha_{c}^{0}=\pi$. This means that one needs a stronger coupling to form a nontrivial $T$ type function. If the coupling is in the region $\frac{\pi}{3}<\alpha<\pi$, only $G_{S+P}$ will have a nontrivial solution, while $G_{T}$ still remains zero; at $\alpha=\pi$ this $G_{T}$ might become nonzero as well. However, one should keep in mind that these $G_{S+P}$ and $G_{T}$ type function are not identical to the four-fermion functions $\mathcal{G}_{S+P}$ and $\mathcal{G}_{T}$ itself, see Eqs. (4.4)-(4.6). So as soon as either $G_{S+P}$ (or $G_{T}$ ) becomes nonzero, both the $S+P$ and the $T$ type nonperturbative four-point functions $\mathcal{G}_{S+P}$ and $\mathcal{G}_{T}$ will become nonzero.

\section{DISCUSSION}

We have shown that, in leading order, both the $S+P$ and the $T$ type four-fermion functions and its condensates are zero below the critical coupling $\alpha_{c}=\frac{\pi}{3}$ in the massless

\footnotetext{
${ }^{3}$ The reason to put the factor $l-1$ in Eq. (4.64) is to keep the equations finite in the case of $l=1$, in which case $b^{\prime}=1$ is a solution. We find in this case that in fact $\frac{l-1}{b^{\prime}-1}=\frac{1}{1+\frac{\alpha}{12 \pi}}$.
} 
fermion phase of a $U(1)$ gauge theory. Above this critical coupling, there can be a nontrivial solution for these nonperturbative chiral-symmetry breaking four-point functions. This result is found using an expansion of the effective action, assuming that the other nonperturbative four-fermion functions (which are not parity or Lorentz invariant) are zero. It agrees with the result found by Holdom and Triantaphyllou [2], who used a truncation of the Schwinger-Dyson equation for the four-fermion functions.

Our result shows that the critical coupling for chiral symmetry breaking by four-fermion condensates is the same as that for dynamical mass generation. Therefore, in a strongly coupled abelian gauge theory, beyond the critical coupling, the chiral symmetry will be broken through the two-fermion condensate since this is energetically favored. This means that in this theory, there is no hierarchy of chiral symmetry breaking, first by the fourfermion condensates, while the fermions remain massless, and another phase transition, at a lower energy, where the fermions acquire a mass.

The generalization to the $N_{f}$ flavor case is straightforward, and does not change our detailed calculations. The only thing we need to do is to label every $G_{i}$ function with flavor indices. There are no flavor mixing effects in the equations, and the flavor symmetry is not believed to be broken dynamically, so the Green's functions for the different flavor indices decouple and the form of the equations is completely same as the ones we discussed. This means that in a $U_{L}\left(N_{f}\right) \otimes U_{R}\left(N_{f}\right)$ gauge theory it seems to be impossible to use fourfermion functions or its condensates to substitute the two-fermion condensate as the chiral order parameter.

In a more complicated theory like a chiral gauge theory, as suggested in [6], there exists in principle the possibility that chiral symmetry is broken through the forming of fourfermion condensates while the fermions remain massless. For such a scenario to be true, there should be some additional symmetry to prevent the fermions from becoming massive. Whether such a hierarchy of chiral symmetry breaking can indeed be realized in a chiral gauge theory, remains an open question; we only showed that in a $U(1)$ gauge theory the critical coupling for the four-fermion condensates is the same as that for the two-fermion condensates.

A similar result has been obtained using a truncation of the Schwinger-Dyson equation for the four-fermion functions [2]. However, the problem with the set of Schwinger-Dyson equations is that it forms an infinite set of coupled equations, and it is not at all obvious how to truncate this set of equations in a consistent way. Using an approximation which is similar to the quenched ladder approximation for the fermion propagator Schwinger-Dyson equation, Holdom and Triantaphyllou have shown numerically that the critical coupling for chiral symmetry breaking by four-fermion condensates is almost the same as that for dynamical mass generation. It is remarkable that both their calculation and our analytical calculation give the same result (the same critical coupling), although the set of Feynman diagrams included in their and our truncation is not the same. This indicates that the essential diagrams are included in both schemes, and that the exact details of the truncation are less important. Of course, this should be confirmed by a study of the next-to-leading order effective potential, or a more accurate truncation scheme for the Schwinger-Dyson equation. We should also consider alternative formulations for the usual effective action, since there are different ways to introduce the auxiliary fields, which might lead to a different result. 


\section{ACKNOWLEDGEMENT}

We would like to thank Koichi Yamawaki, Yoonbai Kim, and Bob Holdom for useful and stimulating discussions. This work has been supported in part by the Nishina Memorial Fund of Japan, the Natural Science Fund of China, and the Japanese Society for the Promotion of Science.

\section{APPENDIX A: ANGULAR INTEGRATION}

In this appendix we discuss the angular integration. Consider the case with three independent momenta, and choose the axes (in Euclidean space) to be

$$
\begin{aligned}
\vec{p}_{1} & =p_{1} \vec{n}_{1} \\
\vec{p}_{2} & =p_{2}\left(\vec{n}_{1} \cos \theta_{p}+\vec{n}_{2} \sin \theta_{p}\right) \\
\vec{q} & =q\left(\vec{n}_{1} \cos \theta_{q_{1}}+\vec{n}_{2} \sin \theta_{q_{1}} \cos \theta_{q_{2}}+\vec{n}_{3} \sin \theta_{q_{1}} \sin \theta_{q_{2}} \cos \phi_{q}+\vec{n}_{4} \sin \theta_{q_{1}} \sin \theta_{q_{2}} \sin \phi_{q}\right),
\end{aligned}
$$

where we use the notation $\vec{p}$ for a four-dimensional vector and $p$ for its absolute value. The angular integration we are interested in is

$$
\begin{aligned}
& \int d \Omega_{q} \frac{\left(\vec{q} \cdot \vec{p}_{2}\right)^{n}}{\left(\vec{q}-\vec{p}_{1}\right)^{2}}= \\
& =\frac{1}{\pi} \int_{0}^{\pi} d \theta_{q_{1}} \int_{0}^{\pi} d \theta_{q_{2}} \sin ^{2} \theta_{q_{1}} \sin \theta_{q_{2}} \frac{q^{n} p_{2}^{n}\left(\cos \theta_{p} \cos \theta_{q_{1}}+\sin \theta_{p} \sin \theta_{q_{1}} \cos \theta_{q_{2}}\right)^{n}}{q^{2}+p_{1}^{2}-2 q p_{1} \cos \theta_{q_{1}}} \\
& =\frac{q^{n} p_{2}^{n}}{2^{n+3} \pi(n+1) \sin \theta_{p}} \oint_{\text {unit circle }} d t\left(1-t^{2}\right) t^{-n-2} \frac{\left(t^{2} e^{-i \theta_{p}}+e^{i \theta_{p}}\right)^{n+1}-\left(t^{2} e^{i \theta_{p}}+e^{-i \theta_{p}}\right)^{n+1}}{\left(q^{2}+p_{1}^{2}\right) t-q p_{1}\left(1+t^{2}\right)} \\
& =I_{n, 0}+I_{n, 1}
\end{aligned}
$$

where the last integration is performed along the unit circle in the complex $t$ plane. There are two poles inside the circle, one is zero and the other is $t_{ \pm}$, which is defined as

$$
t_{ \pm} \equiv \frac{p_{1}^{2}+q^{2} \pm \sqrt{\left(p_{1}^{2}+q^{2}\right)^{2}-4 p_{1}^{2} q^{2}}}{2 p_{1} q}=\left\{\frac{\min \left[p_{1}, q\right]}{\max \left[p_{1}, q\right]}, \frac{\max \left[p_{1}, q\right]}{\min \left[p_{1}, q\right]}\right\} .
$$

Their contributions to the integration are denoted by $I_{n, 0}$ and $I_{n, 1}$ respectively. We further find

$$
\begin{aligned}
I_{n, 0}= & -\frac{q^{n-1} p_{2}^{n}}{2^{n+1}(n+1) p_{1} \sin \theta_{p}}\left\{\sum _ { k = 0 , n > 0 } ^ { [ \frac { n - 1 } { 2 } ] } C _ { n + 1 } ^ { k } \operatorname { s i n } [ ( n + 1 - 2 k ) \theta _ { p } ] \left[U_{n-1-2 k}\left(\frac{p_{1}^{2}+q^{2}}{2 p_{1} q}\right)\right.\right. \\
& \left.\left.-U_{n+1-2 k}\left(\frac{p_{1}^{2}+q^{2}}{2 p_{1} q}\right)\right]-\sum_{k=\left[\frac{n}{2}\right]}^{\left[\frac{n+1}{2}\right]} C_{n+1}^{k} \sin \left[(n+1-2 k) \theta_{p}\right] U_{n+1-2 k}\left(\frac{p_{1}^{2}+q^{2}}{2 p_{1} q}\right)\right\}, \\
I_{n, 1}= & -\frac{q^{n-1} p_{2}^{n}}{2^{n+1}(n+1) p_{1} \sin \theta_{p}} \sum_{k=0}^{n+1} C_{n+1}^{k} t_{-}^{-n-1+2 k} \sin \left[(n+1-2 k) \theta_{p}\right],
\end{aligned}
$$

where we have used the standard Chebyshev expansion [17] 


$$
\frac{1}{1-\frac{p_{1}^{2}+q^{2}}{p_{1} q} t+t^{2}}=\sum_{n=0}^{\infty} t^{n} U_{n}\left(\frac{p_{1}^{2}+q^{2}}{2 p_{1} q}\right) .
$$

Using another property of the Chebyshev functions

$$
\sin [(n+1) \theta]=U_{n}(\cos \theta) \sin \theta,
$$

the result of Eq. (A2) can be further expressed as

$$
\begin{aligned}
\int d \Omega_{q} \frac{\left(\vec{q} \cdot \vec{p}_{2}\right)^{2 n}}{\left(q-p_{1}\right)^{2}} & =\frac{q^{2 n-1} p_{2}^{2 n}}{2^{2 n+1}(2 n+1) p_{1}} \sum_{k=0}^{n} C_{2 n+1}^{n-k} U_{2 k}\left(\cos \theta_{p}\right) X_{2 k}\left(\frac{p_{1}^{2}+q^{2}}{2 p_{1} q}\right), \\
\int d \Omega_{q} \frac{\left(\vec{q} \cdot \vec{p}_{2}\right)^{2 n+1}}{\left(q-p_{1}\right)^{2}} & =\frac{q^{2 n} p_{2}^{2 n+1}}{2^{2 n+2}(2 n+2) p_{1}} \sum_{k=1}^{n+1} C_{2 n+2}^{n+1-k} U_{2 k-1}\left(\cos \theta_{p}\right) X_{2 k-1}\left(\frac{p_{1}^{2}+q^{2}}{2 p_{1} q}\right),
\end{aligned}
$$

where

$$
X_{n}(x) \equiv U_{n+1}(x)-U_{n-1}(x)+\left(x-\sqrt{x^{2}-1}\right)^{n+1}-\left(x+\sqrt{x^{2}-1}\right)^{n+1} .
$$

To calculate $X_{n}(x)$, we consider the quantity

$$
\begin{aligned}
\sum_{n=0}^{\infty} t^{n} X_{n}(x) & =\sum_{n=0}^{\infty} t^{n}\left[U_{n+1}(x)-U_{n-1}(x)+\left(x-\sqrt{x^{2}-1}\right)^{n+1}-\left(x+\sqrt{x^{2}-1}\right)^{n+1}\right] \\
& =\frac{1}{t}\left[-1+\frac{1-t^{2}-2 t \sqrt{x^{2}-1}}{1-2 x t+t^{2}}\right] \\
& =2 \frac{x-\sqrt{x^{2}-1}}{1-t\left(x-\sqrt{x^{2}-1}\right)} \\
& =2 \sum_{n=0}^{\infty}\left(x-\sqrt{x^{2}-1}\right)^{n+1} t^{n}
\end{aligned}
$$

where Eq. (A6) is used. Comparing both sides of the above equation, we find

$$
X_{n}(x)=2\left(x-\sqrt{x^{2}-1}\right)^{n+1} .
$$

Now use the definition of the Chebyshev polynomials [17]

$$
U_{n}(x)=\sum_{k=0}^{\left[\frac{n}{2}\right]}(-1)^{k} \frac{(n-k) !}{k !(n-2 k) !}(2 x)^{n-2 k},
$$

to obtain

$$
\begin{gathered}
\int d \Omega_{q} \frac{U_{2 n}\left(\frac{\vec{q} \cdot \vec{p}_{2}}{q p_{2}}\right)}{\left(q-p_{1}\right)^{2}}=\frac{(-1)^{n}}{p_{1} q} \sum_{k=0}^{n} \alpha_{k}^{(2 n)} U_{2 k}\left(\frac{\vec{p}_{1} \cdot \vec{p}_{2}}{p_{1} p_{2}}\right) t_{-}^{2 k+1}, \\
\int d \Omega_{q} \frac{U_{2 n+1}\left(\frac{\vec{q} \cdot \vec{p}_{2}}{q p_{2}}\right)}{\left(q-p_{1}\right)^{2}}=\frac{(-1)^{n}}{p_{1} q} \sum_{k=0}^{n} \alpha_{k}^{(2 n+1)} U_{2 k+1}\left(\frac{\vec{p}_{1} \cdot \vec{p}_{2}}{p_{1} p_{2}}\right) t_{-}^{2 k+2},
\end{gathered}
$$

in which $\alpha_{k}^{(2 n)}$ and $\alpha_{k}^{(2 n+1)}$ are defined as 


$$
\begin{aligned}
\alpha_{k}^{(2 n)} & \equiv \sum_{m=k}^{n}(-1)^{m} \frac{(n+m) !}{(n-m) !(m-k) !(m+1+k) !} \\
\alpha_{k}^{(2 n+1)} & \equiv \sum_{m=k}^{n}(-1)^{m} \frac{(n+m+1) !}{(n-m) !(m-k) !(m+2+k) !}
\end{aligned}
$$

Finally, to calculate the coefficients $\alpha$, we introduce two functions

$$
\begin{aligned}
& F_{n, k}(x) \equiv \sum_{m=k}^{n}(-1)^{m} \frac{(n+m+2 k) !}{(n-m) ! m !(m+1+2 k) !} x^{m}, \\
& \bar{F}_{n, k}(x) \equiv \sum_{m=k}^{n}(-1)^{m} \frac{(n+m+2 k+1) !}{(n-m) ! m !(m+2+2 k) !} x^{m}
\end{aligned}
$$

so we get

$$
\begin{aligned}
\alpha_{k}^{(2 n)} & =(-1)^{k} F_{n-k, k}(1), \\
\alpha_{k}^{(2 n+1)} & =(-1)^{k} \bar{F}_{n-k, k}(1) .
\end{aligned}
$$

For $n \leq 1$ we find

$$
\begin{aligned}
F_{0, k}(x) & =\frac{1}{2 k+1}, \\
\bar{F}_{0, k}(x) & =\frac{1}{2 k+2}, \\
F_{1, k}(x) & =1-x, \\
\bar{F}_{1, k}(x) & =1-x,
\end{aligned}
$$

whereas for $n>1$

$$
\begin{aligned}
& F_{n, k}(x)=\frac{1}{n !} x^{-2 k-1} \frac{d^{n-1}}{d x^{n-1}}\left[x^{2 k+n}(1-x)^{n}\right], \\
& \bar{F}_{n, k}(x)=\frac{1}{n !} x^{-2 k-2} \frac{d^{n-1}}{d x^{n-1}}\left[x^{2 k+n+1}(1-x)^{n}\right],
\end{aligned}
$$

so the total result can be written as

$$
\begin{aligned}
F_{n, k}(1) & =\frac{\delta_{n 0}}{2 k+1}, \\
\bar{F}_{n, k}(1) & =\frac{\delta_{n 0}}{2 k+2}, \\
\alpha_{k}^{(2 n)} & =\frac{(-1)^{n}}{2 n+1} \delta_{n k}, \\
\alpha_{k}^{(2 n+1)} & =\frac{(-1)^{n}}{2 n+2} \delta_{n k},
\end{aligned}
$$

Substituting this result into Eqs. (A14) and (A15), we finally get the result

$$
\int d \Omega_{q} \frac{U_{n}\left(\hat{q} \cdot \hat{p}_{2}\right)}{\left(\vec{q}-\vec{p}_{1}\right)^{2}}=\frac{1}{(n+1) p_{1} q}\left[\frac{\min \left[p_{1}, q\right]}{\max \left[p_{1}, q\right]}\right]^{n+1} U_{n}\left(\hat{p}_{1} \cdot \hat{p}_{2}\right),
$$


where $\hat{p} \equiv \vec{p} / p$ denotes the angular dependence.

Next, we introduce a "generalized Chebyshev function" with Lorentz indices $\mu_{1} \ldots \mu_{n}$ by extracting the $\hat{p}$ dependence in $U_{n}(\hat{q} \cdot \hat{p})$

$$
U_{n}(\hat{q} \cdot \hat{p}) \equiv \hat{p}_{\mu_{1}} \ldots \hat{p}_{\mu_{n}} \hat{U}_{n}^{\mu_{1} \ldots \mu_{n}}(\hat{q})
$$

which defines the generalized Chebyshev functions $\hat{U}_{n}^{\mu_{1} \ldots \mu_{n}}$ as

$$
\begin{aligned}
\hat{U}_{2 n}^{\mu_{1} \ldots \mu_{2 n}}(\hat{q})= & \sum_{k=0}^{n} \frac{(-1)^{k} 2^{2 n-2 k}(2 n-k) !}{k !(2 n-2 k) !(2 n) !}\left[\hat{q}^{\mu_{1}} \ldots \hat{q}^{\mu_{2 n-2 k}} \delta^{\mu_{2 n-2 k+1} \mu_{2 n-2 k+2}} \ldots \delta^{\mu_{2 n-1} \mu_{2 n}}\right. \\
& \left.+ \text { all symmetric permutations in }\left(\mu_{1} \ldots \mu_{2 n}\right)\right], \\
\hat{U}_{2 n+1}^{\mu_{1} \ldots \mu_{2 n+1}}(\hat{q})= & \sum_{k=0}^{n} \frac{(-1)^{k} 2^{2 n+1-2 k}(2 n+1-k) !}{k !(2 n+1-2 k) !(2 n+1) !}\left[\hat{q}^{\mu_{1}} \ldots \hat{q}^{\mu_{2 n+1-2 k}} \delta^{\mu_{2 n+2-2 k} \mu_{2 n+3-2 k}} \ldots \delta^{\mu_{2 n} \mu_{2 n+1}}\right. \\
& \left.+ \text { all symmetric permutations in }\left(\mu_{1} \ldots \mu_{2 n+1}\right)\right] .
\end{aligned}
$$

Since in Eq. (A32), $p_{2}$ is an arbitrary momentum variable, we can use the expansion Eq. (A33) to find

$$
\int d \Omega_{q} \frac{\hat{U}_{n}^{\mu_{1} \ldots \mu_{n}}(\hat{q})}{\left(\vec{q}-\vec{p}_{1}\right)^{2}}=\frac{1}{(n+1) p_{1} q}\left[\frac{\min \left[p_{1}, q\right]}{\max \left[p_{1}, q\right]}\right]^{n+1} \hat{U}_{n}^{\mu_{1} \ldots \mu_{n}}\left(\hat{p}_{1}\right) .
$$

This formula allows us to perform all of the angular integration which are needed, rest us to show that we can indeed expand our four-point functions in terms of these generalized Chebyshev functions. The Chebyshev polynomials $U_{n}(x)$ form a complete set of independent functions, so from Eq. (A33), it follows immediately that our generalized Chebyshev functions are also a complete set for functions which are totally symmetric in their Lorentz indices. The explicit expression of this statement can be seen from following identities, noting that

$$
\begin{aligned}
(\hat{p} \cdot \hat{q})^{2 n} & =\sum_{k=0}^{n} c_{n, k} U_{2 k}(\hat{p} \cdot \hat{q}) \\
& =p_{\mu_{1}} \ldots p_{\mu_{2 n}} \sum_{k=0}^{n} c_{n, k} \hat{U}_{2 k}^{\mu_{1} \ldots \mu_{2 k}}(\hat{q}) \delta^{\mu_{2 k+1} \mu_{2 k+2}} \ldots \delta^{\mu_{2 n-1} \mu_{2 n}} \\
(\hat{p} \cdot \hat{q})^{2 n+1} & =\sum_{k=0}^{n} c_{n, k}^{\prime} U_{2 k+1}(\hat{p} \cdot \hat{q}) \\
& =p_{\mu_{1}} \ldots p_{\mu_{2 n+1}} \sum_{k=0}^{n} c_{n, k}^{\prime} \hat{U}_{2 k+1}^{\mu_{1} \ldots \mu_{2 k+1}}(\hat{q}) \delta^{\mu_{2 k+2} \mu_{2 k+3}} \ldots \delta^{\mu_{2 n} \mu_{2 n+1}}
\end{aligned}
$$

which follows directly from Eq. (A33) and the fact the Chebyshev polynomials form a complete set. Now due to the fact that $\hat{p}$ is arbitrary, the Eqs. (A37) and (A38) are equivalent to

$$
\hat{q}^{\mu_{1}} \ldots \hat{q}^{\mu_{2 n}}=\sum_{k=0}^{n} \frac{c_{n, k}}{(2 n) !}\left[\hat{U}_{2 k}^{\mu_{1} \ldots \mu_{2 k}}(\hat{q}) \delta^{\mu_{2 k+1} \mu_{2 k+2}} \ldots \delta^{\mu_{2 n-1} \mu_{2 n}}\right.
$$




$$
\begin{array}{r}
\left.+ \text { all symmetric permutations in }\left(\mu_{1} \ldots \mu_{2 n}\right)\right] \\
\hat{q}^{\mu_{1}} \ldots \hat{q}^{\mu_{2 n+1}}=\sum_{k=0}^{n} \frac{c_{n, k}^{\prime}}{(2 n+1) !}\left[\hat{U}_{2 k+1}^{\mu_{1} \ldots \mu_{2 k+1}}(\hat{q}) \delta^{\mu_{2 k+2} \mu_{2 k+3}} \ldots \delta^{\mu_{2 n} \mu_{2 n+1}}\right. \\
\left.+ \text { all symmetric permutations in }\left(\mu_{1} \ldots \mu_{2 n+1}\right)\right] .
\end{array}
$$

So all $\hat{q}^{\mu_{1}} \ldots \hat{q}^{\mu_{n}}$ can be expanded in terms of our generalized Chebyshev polynomials. Since any smooth function of $\vec{q}$ can always be expanded in terms of $\hat{q}^{\mu_{1}} \ldots \hat{q}^{\mu_{n}}$ times a function which depends only on the absolute value $q$, this means that we can always expand our four-point functions in terms of these Chebyshev functions. 


\section{REFERENCES}

[1] V.A. Miransky, Dynamical Symmetry Breaking in Quantum Field Theories, World Scientific, Singapore, 1993.

[2] B. Holdom and G. Triantaphyllou, Phys. Rev. D 51, 7124 (1995).

[3] B. Holdom and G. Triantaphyllou, Chiral Symmetry Breaking via Multi-fermion Green Functions, UTPT-95-10, hep-ph/9505364.

[4] K. Yamawaki and T. Yokota, Phys. Lett. 113B, 293 (1982).

[5] B. Holdom, Phys. Lett. B 336, 85 (1994).

[6] B. Holdom, Four Fermion Dynamics and Fermion Masses, UTPT-95-22, hepph/9510249, to appear in the proceedings of the Yukawa International Seminar '95: From the Standard Model to Grand Unified Theories, Kyoto, Japan, 21-25 August 1995.

[7] C. Vafa and E. Witten, Nucl. Phys. B234, 173 (1984).

[8] C. Vafa and E. Witten, Phys. Rev. Lett 53, 535 (1984).

[9] Y. Nambu, Phys. Rev. Lett. 4, 380 (1960); Y. Nambu and G. Jona-Lasinio, Phys. Rev. 112, 345 (1961).

[10] J. Goldstone, Nuovo Cimento 19, 154 (1961); J. Goldstone, A. Salam, and S. Weinberg, Phys. Rev. 127, 965 (1962).

[11] Y. Nambu, Phys. Lett. 26B, 626 (1966); S. Coleman and E. Weinberg, Phys. Rev D 7, 1888 (1973).

[12] R. Jackiw, Phys. Rev. D 9, 1686 (1974).

[13] J.M. Cornwall, R. Jackiw and E.Tomboulis, Phys. Rev. D 10, 2428 (1974).

[14] T. Maskawa and H. Nakajima, Prog. Theor. Phys. 52, 1326 (1974).

[15] P.I. Fomin and V.A. Miransky, Phys. Lett. B64, 166 (1976).

[16] P.I. Fomin, V.P. Gusynin, V.A. Miransky, and Yu.A. Sitenko, Riv. Nuovo Cimento 6, $1(1983)$.

[17] M. Abramowitz and I.A. Stegun, editors, Handbook of Mathematical Functions, Dover Publications, New York, 1965. 


\section{FIGURES}

FIG. 1. Our truncated equation for the nonperturbative four-fermion function (diagrammati-

cally): (a) $\mathcal{G}\left(p_{1}, p_{2}, p_{3}, p_{4}\right)$ decomposed into the $s$ - and $t$-channel components $G\left(p_{1}, p_{2}, p_{3}, p_{4}\right)$; (b) the equation for the $s$-channel component; and (c) the equation for the $t$-channel. 

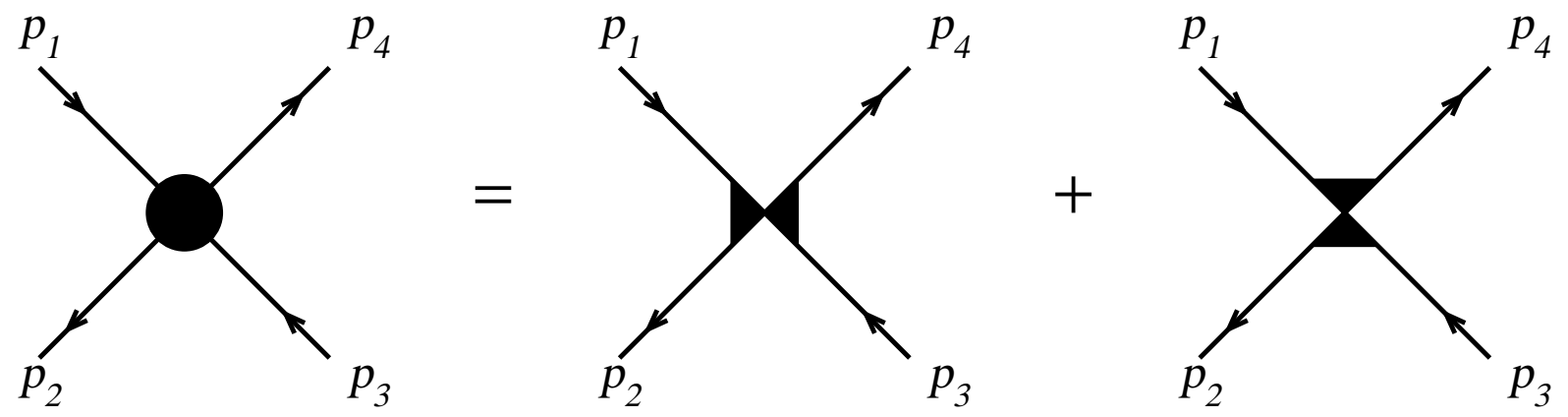

Figure 1 (a)
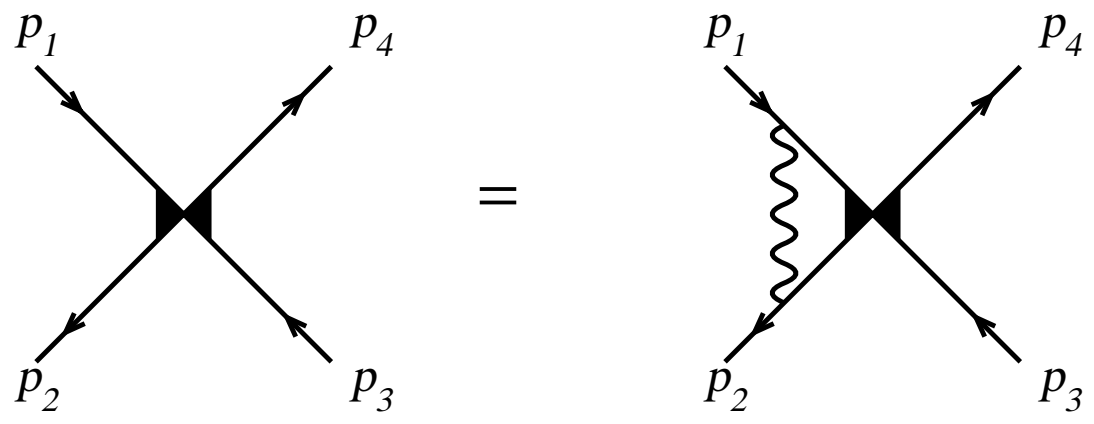

Figure 1 (b)
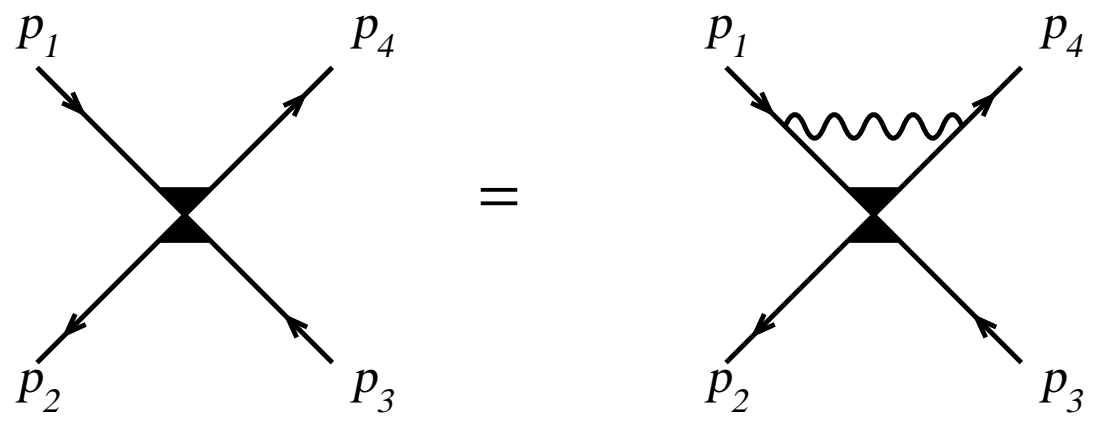

Figure 1 (c) 